\title{
Bi-Hamiltonian operators, integrable flows of curves using moving frames, and geometric map equations
}

\author{
Stephen C. Anco \\ Department of Mathematics \\ Brock University, St. Catharines, ON Canada \\ E-mail: sanco@brocku.ca
}

\begin{abstract}
Moving frames of various kinds are used to derive bi-Hamiltonian operators and associated hierarchies of multi-component soliton equations from groupinvariant flows of non-stretching curves in constant curvature manifolds and Lie group manifolds. The hierarchy in the constant-curvature case consists of a vector $\mathrm{mKdV}$ equation coming from a parallel frame, a vector potential mKdV equation coming from a covariantly-constant frame, and higher order counterparts generated by an underlying vector $\mathrm{mKdV}$ recursion operator. In the Lie-group case the hierarchy comprises a group-invariant analog of the vector NLS equation coming from a left-invariant frame, along with higher order counterparts generated by a recursion operator that is like a square-root of the $\mathrm{mKdV}$ one. The corresponding respective curve flows are found to be given by geometric nonlinear PDEs, specifically $\mathrm{mKdV}$ and group-invariant analogs of Schrödinger maps. In all cases the hierarchies also contain variants of vector sineGordon equations arising from the kernel of the respective recursion operators. The geometric PDEs that describe the corresponding curve flows are shown to be wave maps. Full details of these results are presented for two main cases: $S^{2}, S^{3} \simeq S U(2)$.
\end{abstract}




\section{Introduction}

Over the years there has been much interest in the close connection between classical soliton equations such as Korteweg de Vries (KdV), nonlinear Schrödinger (NLS), sine-Gordon (SG), on one hand, and nonlinear geometric partial differential equations (PDEs) on the other hand, particularly wave maps and Schrödinger maps which are natural generalizations of the ordinary wave equation and Schrödinger equation in $1+1$ dimensions for a function taking values in a Riemannian or Kähler target space $N$.

One prominent example is the $O(3)$ Heisenberg model $\vec{u}_{t}=\vec{u} \times \vec{u}_{x x},|\vec{u}|=1$, which is geometrically just a Schrödinger map equation $\gamma_{t}=J \nabla_{x} \gamma_{x}$ where $\gamma(t, x)$ is a map into the target space $S^{2}$, and $\nabla_{x}, J$ are the pull back of the covariant derivative and the complex structure tensor (Hodge-star operator) determined by the metric on $S^{2}$ in terms of the local coordinates represented by $\gamma$. This model is well known to be equivalent to the NLS equation $0=\mathrm{i} \phi_{t}+\phi_{x x}+2|\phi|^{2} \phi$ by means of a transformation [1] found by Hasimoto. $\ddagger$ The transformation is $\phi=\kappa \exp \left(\mathrm{i} \int \tau d x\right)$ where $\kappa, \tau$ are the curvature and torsion associated to $\vec{u}$ viewed as the tangent vector to an arclength-parametrized space curve in $\mathbb{R}^{3}$, whose Euclidean Frenet frame is $(\vec{T}, \vec{N}, \vec{B})=\left(\vec{u}, \kappa^{-1} \vec{u}_{x}, \kappa^{-1} \vec{u} \times \vec{u}_{x}\right)$, evolving under the Heisenberg equation. This evolution gives the equations $\kappa_{t}=-2 \tau \kappa_{x}-\kappa \tau_{x}$, $\tau_{t}=\kappa^{-1} \kappa_{x x x}-\kappa^{-2} \kappa_{x} \kappa_{x x}+\kappa \kappa_{x}-2 \tau \tau_{x}$, coinciding with the motion of a vortex filament studied by Hasimoto [3]. A more direct geometrical formulation of the Hasimoto transformation has been obtained in the context of recent analytical work [4] on the Cauchy problem for $S^{2}$ Schrödinger maps. The main idea is to introduce a covariantlyconstant orthonormal frame $e_{1}, e_{2}=J e_{1}$ satisfying $\nabla_{x} e_{1}=\nabla_{x} e_{2}=0$ along the curve in $S^{2}$ defined by $\gamma(t, x)$ for $t=$ const. The evolution of the components of the tangent vector $\gamma_{x}=q_{1} e_{1}+q_{2} e_{2}$ in this frame then reduces precisely to the NLS equation for $\phi=q_{1}+\mathrm{i} q_{2}$.

Another example of wide interest is the $O(3)$ sigma model $\vec{u}_{t x}=-\vec{u} \vec{u}_{t} \cdot \vec{u}_{x},|\vec{u}|=1$. This model has long been known to be equivalent [5] to the $\mathrm{SG}$ equation $0=\theta_{t x}+\sin \theta$ through the geometrical relation $\vec{u}_{t} \cdot \vec{u}_{x}=\left|\vec{u}_{t}\right|\left|\vec{u}_{x}\right| \cos \theta$ combined with a conformal scaling of $t, x$, such that $\left|\vec{u}_{t}\right|=\left|\vec{u}_{x}\right|=1$ as allowed by the conservation laws $D_{x}\left|\vec{u}_{t}\right|=D_{t}\left|\vec{u}_{x}\right|=0$. The equivalence can be looked on as a Hasimoto transformation [6] $\theta=-\int \tau d x$ in terms of the torsion $\tau$ given by $\vec{u}$ which is again viewed as determining a Euclidean Frenet frame $(\vec{T}, \vec{N}, \vec{B})=\left(\vec{u}, \kappa^{-1} \vec{u}_{x}, \kappa^{-1} \vec{u} \times \vec{u}_{x}\right)$ of an arclength-parameterized space curve in $\mathbb{R}^{3}$ but now evolving under the sigma model equation and subject to constant curvature $\kappa=1$. The equations for the evolution of the torsion and curvature are simply $\kappa_{t}=0, \tau_{t x}=-\tau \sqrt{1-\tau_{t}^{2}}$. A deeper origin for the SG equation comes from the geometric formulation of the $O(3)$ sigma model as an $S^{2}$ wave map equation $\nabla_{x} \gamma_{t}=0$ where $\gamma(t, x)$ is again a map into the target space $S^{2}$. The curve for $t=$ const defined by this map $\gamma(t, x)$ moves with uniform speed and does not stretch, because of the previous conservation laws. Consequently, a conformal scaling of $t, x$ allows a covariantly-constant orthonormal frame $e_{1}, e_{2}$ to be adapted to the evolution vector $\ddagger$ In fact, the Hasimoto transformation was already known at the start of the last century [2]. 
$\gamma_{t}=e_{1}$, with $\nabla_{x} e_{1}=\nabla_{x} e_{2}=0$ holding along the curve. The tangent vector then takes the form $\gamma_{x}=\cos \theta e_{1}+\sin \theta e_{2}$ in terms of a rotation angle $\theta$ whose evolution is just given by the $\mathrm{SG}$ equation.

From a closely related point of view, the mKdV equation is known to arise [7] as an $O(3)$-invariant motion of a non-stretching curve $\gamma(t, x)$ in $S^{2}$ as formulated using a moving orthonormal frame adapted to the curve so that $\gamma_{x}=\kappa e_{1}, J \gamma_{x}=\kappa e_{2}$, (i.e. an intrinsic Frenet frame) where $\kappa_{t}=0$ is the condition of no stretching. $O(3)$ invariance means that the frame components of the motion $\gamma_{t}=h_{1} e_{1}+h_{2} e_{2}$ must depend only on differential invariants of the curve under the group action determined by the isometry group $O(3)$ of $S^{2}$. There is a fundamental invariant given by the intrinsic curvature of $\gamma(t, x)$ defined through the Serret-Frenet equations on $S^{2}$ for the moving frame $\nabla_{x} e_{1}=\tau e_{2}, \nabla_{x} e_{2}=-\tau e_{1}$, with $x$ conformally scaled proportional to the arclength, so $\kappa=$ const. This invariant $\tau$ also can be viewed as the torsion of an arclengthparameterized space curve in $\mathbb{R}^{3}$ whose Euclidean Frenet frame is associated to $\gamma(t, x)$ via the identifications $\gamma \mapsto \vec{T}=\vec{u}, e_{1} \mapsto \vec{N}=\kappa^{-1} \vec{u}_{x}, e_{2} \mapsto \vec{B}=\kappa^{-1} \vec{u} \times \vec{u}_{x}$ under the embedding of $S^{2}$ into $\mathbb{R}^{3}$ given by $|\vec{u}|=1$. Since the curvature of this space curve in $\mathbb{R}^{3}$ is $\kappa=$ const, a complete set of differential invariants is obtained from $x$-derivatives of the torsion $\tau$, and thus $h_{1}=h_{1}\left(\tau, \tau_{x}, \tau_{x x}, \ldots\right), h_{2}=h_{2}\left(\tau, \tau_{x}, \tau_{x x}, \ldots\right)$. The nonstretching condition on the motion of the curve imposes the relation $D_{x} h_{1}=\tau h_{2}$, while the equation of motion of the curve yields the flow $\tau_{t}=\kappa^{-1} D_{x}\left(D_{x} h_{2}+\tau h_{1}\right)+\kappa h_{2}$. For the particular motion $h_{1}=\frac{1}{2} \tau^{2}-\kappa^{2}, h_{2}=\tau_{x}$, this flow becomes precisely the mKdV equation $\tau_{t}=\kappa^{-1}\left(\tau_{x x x}+\frac{3}{2} \tau^{2} \tau_{x}\right)$ on $\tau$. More remarkably, as shown in Ref. [7, when the flow of a non-stretching curve is expressed in the operator form $\tau_{t}=\kappa^{-1} \mathcal{R}\left(h_{2}\right)+\kappa h_{2}$ with $h_{1}=D_{x}^{-1}\left(\tau h_{2}\right)$ put in terms of the normal frame component $h_{2}$ of the evolution vector, it contains the recursion operator $\mathcal{R}=D_{x}^{2}+\tau^{2}+\tau_{x} D_{x}^{-1} \tau$ of the hierarchy of $\mathrm{mKdV}$ soliton equations. For instance, the 5 th order $\mathrm{mKdV}$ equation on $\tau$ arises from the curve motion generated by $h_{2}=\mathcal{R}\left(\tau_{x}\right)-\kappa^{2} \tau_{x}=\tau_{x x x}+\left(\frac{3}{2} \tau^{2}-\kappa^{2}\right) \tau_{x}$, $h_{1}=D_{x}^{-1}\left(\tau \mathcal{R}\left(\tau_{x}\right)-\kappa^{2} \tau \tau_{x}\right)=\tau \tau_{x x}-\frac{1}{2} \tau_{x}^{2}+\frac{3}{8} \tau^{4}-\frac{1}{2} \kappa^{2} \tau^{2}$.

More generally, other recent work has studied group-invariant motions of nonstretching curves $\gamma(t, x)$ in higher dimensional constant curvature spaces $N \simeq$ $S^{n}, H^{n}, \mathbb{R}^{n}$, first in $n=3$ dimensions [8] and subsequently in all dimensions $n \geq 2$ [9]. The main results give a geometric origin of the vector mKdV equation $\boldsymbol{v}_{t}=$ $\boldsymbol{v}_{x x x}+\frac{3}{2} \boldsymbol{v} \cdot \boldsymbol{v} \boldsymbol{v}_{x}$ and its associated hierarchy of integrable soliton equations, for an $n-1$ component vector variable $\boldsymbol{v}(t, x)$ given by the frame components of the principal normal along the curve, generalizing the scalar case $n=2$. This generalization relies on the use of a parallel moving (orthonormal) frame adapted to the curve $\gamma(t, x)$, differing from an intrinsic Frenet (orthonormal) frame. In a parallel frame [10] the normal frame vectors are defined to have a purely tangential derivative along the curve, while the derivative of the tangent frame vector is normal to the curve. These properties uniquely determine a Frenet frame in $n=2$ dimensions; in $n>2$ dimensions a parallel frame is related to a Frenet frame by a certain local $S O(n-1)$ rotation acting on the normal vectors, and in the case $n=3[12$ this rotation angle is given by the formula for a Hasimoto 
transformation in terms of the torsion determined by the Serret-Frenet equations of the frame. As shown in Ref. [9, 11], the Cartan structure equations (which generalize the Serret-Frenet equations [13]) for such a frame and its associated connection matrix coming from the flow of a non-stretching curve can be seen to encode compatible Hamiltonian symplectic and cosymplectic operators with respect to the Hamiltonian flow variable $\boldsymbol{v}$; moreover, these operators produce a hereditary recursion operator whenever the flow is invariant under the $S O(n-1)$ structure group preserving the frame. In the case $n=2$, for spaces $N$ with constant Gaussian curvature $\chi$ (i.e. $\chi=+1$ for the sphere $N=S^{2}, \chi=-1$ for the hyperboloid $N=H^{2}, \chi=0$ for the plane $N=\mathbb{R}^{2}$ ), the encoding of the operators is especially simple. Here $\boldsymbol{v}$ is just a 1-component variable identified with the invariant $\tau$ of the curve $\gamma(t, x)$, while $x$ is the arclength so $\kappa=1$. The frame equation of motion of $\gamma(t, x)$ has the Hamiltonian formulation $\tau_{t}=\mathcal{H}(\varpi)+\chi \mathcal{I}(\varpi)$ where $\mathcal{H}, \mathcal{I}$ are compatible mKdV Hamiltonian (cosymplectic) operators, defined by $\varpi=D_{x} h_{\perp}+\tau D_{x}^{-1}\left(\tau h_{\perp}\right)=\mathcal{I}^{-1}\left(h_{\perp}\right)$ which is a Hamiltonian symplectic operator and $D_{x} \varpi=\mathcal{H}(\varpi)$. In geometrical terms, $h_{\perp}$ is the normal frame component of the curve motion $\gamma_{t}$, and $\varpi$ is the associated connection component in the Serret-Frenet equations for this motion, $\nabla_{t} e_{1}=\varpi e_{2}, \nabla_{t} e_{2}=-\varpi e_{1}$. Viewed in a Hamiltonian setting, $h_{\perp} \partial / \partial \tau$ and $\varpi d \tau$ represent a Hamiltonian vector field and covector field on the $x$-jet space of the flow variable $\tau(t, x)$. Because $\mathcal{H}, \mathcal{I}$ are a bi-Hamiltonian pair, $\mathcal{R}=\mathcal{H} \circ \mathcal{I}^{-1}$ is a recursion operator on Hamiltonian vector fields $h_{\perp} \partial / \partial \tau$, while its adjoint $\mathcal{R}^{*}=\mathcal{I}^{-1} \circ \mathcal{H}$ is a recursion operator on covector fields $\varpi d \tau$ with $\varpi=\delta H / \delta \tau$ holding for some Hamiltonian expressions $H=H\left(\tau, \tau_{x}, \tau_{x x}, \ldots\right)$. This structure gives rise to a hierarchy of commuting Hamiltonian mKdV flows on $\tau$, corresponding to integrable non-stretching curve motions in $S^{2}$.

Bringing together all these ideas, the purpose of this paper is, firstly, to identify the geometric "map equations" corresponding to the hierarchy of integrable flows of nonstretching curves in constant curvature spaces and, secondly, to extend this result as well as the geometric relation known so far between the parallel moving frame formulation of these flows and bi-Hamiltonian operators to other kinds of frames and more general target spaces. The main results will be derived in two and three dimensions.

In particular, it is shown that:

- the curve flow in $S^{2}$ corresponding to the scalar mKdV equation in a parallel moving frame is described by an mKdV analog of the Schrödinger map equation;

- in a covariantly-constant moving frame the mKdV curve flow in $S^{2}$ corresponds to the scalar potential $\mathrm{mKdV}$ equation and encodes bi-Hamiltonian operators yielding the $\mathrm{mKdV}$ recursion operator in potential form;

- the kernel of the $\mathrm{mKdV}$ recursion operator gives rise to the SG equation describing a curve flow in $S^{2}$ given by the wave map equation;

- analogous curve flows in $S^{3}$ formulated in both covariantly-constant and parallel moving frames yield bi-Hamiltonian operators associated to $O(2)$-invariant vector generalizations of the potential $\mathrm{mKdV}$ equation and the SG equation;

- an enlarged hierarchy of curve flows in $S^{3}$ arises through a "square-root" of the 
vector mKdV recursion operator derived from an encoding of bi-Hamiltonian operators in a left-invariant moving frame which is tied to the Lie group structure $S U(2) \simeq S^{3}$;

- the bottom flow in the enlarged hierarchy is a group-invariant analog of the Schrödinger map equation.

In addition, higher dimensional generalizations of these geometric map equations, vector soliton equations, and bi-Hamiltonian operators will be obtained for flows of curves in constant curvature spaces via a covariantly-constant moving frame and in Lie group spaces via a left-invariant moving frame. Underlying all these results is a main insight that the torsion and curvature parts of the Cartan structure equations associated to the general flow of a curve as formulated by a suitable moving frame (adapted either to the curve or to the geometry in which the curve moves) together carry a geometrical encoding of bi-Hamiltonian operators.

Related work on derivations of soliton equations from flows of curves in conformal and similarity geometries, affine geometry, and Klein geometries appears in Ref. [14, 11, 15, 16, 17, 18.

Key definitions pertaining to Hamiltonian structures are stated in the appendix. See Ref. [19, 20] for a full summary of Hamiltonian theory developed for PDE systems.

\section{Wave maps and mKdV maps from integrable flows of curves on the sphere and the hyperboloid}

Consider a flow of a non-stretching curve $\gamma(t, x)$ in a two-dimensional constant curvature space: $N=S^{2}$ the sphere, or $N=H^{2}$ the hyperboloid, or $N=\mathbb{R}^{2}$ the plane. Let $\chi$ denote the Gaussian curvature, respectively $+1,-1,0$, let $g$ denote the metric tensor, and let $J$ be the Hodge-star operator. To begin, the flow equation of motion of $\gamma(t, x)$ will be shown to exhibit a natural Hamiltonian structure when formulated in a covariantlyconstant orthonormal frame,

$$
\nabla_{x} e_{1}=\nabla_{x} e_{2}=0, \quad e_{2}=J e_{1} .
$$

First, the non-stretching condition $\left|\gamma_{x}\right|_{g}=1$ with $x$ scaled to be the arclength implies that the tangent vector and normal vector to the curve $\gamma(t, x)$ are given by

$$
\gamma_{x}=\cos \theta e_{1}+\sin \theta e_{2}=X, \quad J \gamma_{x}=\sin \theta e_{1}-\cos \theta e_{2}=J X,
$$

where the variable $\theta$ is a rotation angle relating the covariantly-constant frame to an adapted (Frenet) frame $X, J X$. Note the Serret-Frenet equations become

$$
\nabla_{x} X=\nu J X
$$

which yields the expression

$$
\nu=-\theta_{x}
$$

for the intrinsic curvature of $\gamma(t, x)$ in $N$. Hence $\theta=-\int \nu d x$ is a nonlocal curvature invariant of $\gamma(t, x)$. Due to this relationship, the Hamiltonian structure arising from the covariantly-constant frame equations will be analogous to a potential formulation. In 
this frame a non-stretching curve motion $\gamma_{t}=h_{1} e_{1}+h_{2} e_{2}$ is $O(3)$-invariant if, and only if, $h_{1}=h_{1}\left(\theta, \theta_{x}, \theta_{x x}, \ldots\right)$ and $h_{2}=h_{2}\left(\theta, \theta_{x}, \theta_{x x}, \ldots\right)$ are functions of the (differential) invariants of the curve. An invariant of motion is $\varpi$ coming from the geometrical equation

$$
\nabla_{t} X=\varpi J X
$$

for the evolution of the frame. In terms of $\theta$ the equation of motion for the curve is given by $D_{x}^{-1}$ applied to the flow equation on $\nu$ (cf. section 1$)$,

$$
\nu_{t}=\mathcal{R}\left(h_{\perp}\right)+\chi h_{\perp}
$$

which yields

$$
-\theta_{t}=\mathcal{R}^{*}\left(D_{x}^{-1} h_{\perp}\right)+\chi D_{x}^{-1} h_{\perp}
$$

with

$$
h_{\perp}=h_{1} \sin \theta-h_{2} \cos \theta
$$

and

$$
h_{\|}=h_{1} \cos \theta+h_{2} \sin \theta=-D_{x}^{-1}\left(\theta_{x} h_{\perp}\right) .
$$

Here

$$
\mathcal{R}^{*}=D_{x}^{2}+\nu^{2}-\nu D_{x}^{-1} \nu_{x}
$$

is the adjoint of the $\mathrm{mKdV}$ recursion operator

$$
\mathcal{R}=D_{x}^{2}+\nu^{2}+\nu_{x} D_{x}^{-1} \nu
$$

This flow equation on $\theta$ possesses a Hamiltonian form

$$
-\theta_{t}=\tilde{\mathcal{H}}\left(h_{\perp}\right)+\chi \tilde{\mathcal{I}}\left(h_{\perp}\right)
$$

where

$$
\tilde{\mathcal{H}}\left(h_{\perp}\right)=D_{x} h_{\perp}+\theta_{x} D_{x}^{-1}\left(\theta_{x} h_{\perp}\right)=\varpi, \quad \tilde{\mathcal{I}}\left(h_{\perp}\right)=D_{x}^{-1} h_{\perp}=\varpi^{\prime}
$$

are compatible Hamiltonian cosymplectic operators. Note $\varpi^{\prime}$ is related to $\varpi$ by its inverse image under $\mathcal{R}^{*}$. Compared to the formulation in an adapted frame, here $h_{\perp}$ (normal frame component of motion) and $\varpi$ (connection component for the frame motion) switch roles, so on the $x$-jet space of the flow variable $\theta(t, x), \varpi \partial / \partial \theta$ represents a Hamiltonian vector field (as likewise does $\varpi^{\prime} \partial / \partial \theta$ ) and $h_{\perp} d \theta$ represents a related covector field. A corresponding feature is that the operators $\tilde{\mathcal{I}}$ and $\tilde{\mathcal{H}}$ are respective inverses of the operators $\mathcal{H}$ and $\mathcal{I}$ appearing in the Hamiltonian form of the flow equation on $\nu$ (cf. section 1)

$$
\nu_{t}=\mathcal{H}(\varpi)+\chi \mathcal{I}(\varpi) .
$$

Consequently the recursion operators $\mathcal{R}=\mathcal{H} \circ \mathcal{I}^{-1}$ and $\tilde{\mathcal{R}}=\tilde{\mathcal{H}} \circ \tilde{\mathcal{I}}^{-1}=\mathcal{I}^{-1} \circ \mathcal{H}=\mathcal{R}^{*}$ arising in the flow equations on $\nu$ and $\theta$ are adjoints. These recursion operators generate a hierarchy of commuting Hamiltonian flows on $\theta$ and $\nu$. 
The structure of the hierarchy looks simplest in the planar case, $\chi=0$. The flow equation (2.12) produces a hierarchy of commuting flows $-\theta_{t}=\varpi^{\left({ }^{(k)}\right.}=\tilde{\mathcal{I}}\left(h_{\perp}{ }^{(k)}\right)$ generated by the operator $\tilde{\mathcal{R}}$, with involutive Hamiltonians $H=H^{(k)}$ given by $\delta H / \delta \theta=$ $h_{\perp}{ }^{(k)}$ forming a hierarchy generated through the adjoint operator $\tilde{\mathcal{R}}^{*}, k=0,1,2, \ldots$; equivalently, there is a hierarchy of commuting flows $\nu_{t}=h_{\perp}{ }^{(k)}$ and corresponding involutive Hamiltonians $H=H^{(k)}$ such that $\delta H / \delta \nu=\varpi^{(k)}$, satisfying $h_{\perp}{ }^{(k)}=\mathcal{H}\left(\varpi^{(k)}\right)$, $k=0,1,2, \ldots$, generated by the operators $\mathcal{R}=\tilde{\mathcal{R}}^{*}$ and $\mathcal{R}^{*}=\tilde{\mathcal{R}}$. The relation $\nu=-\theta_{x}$ induces a one-to-one correspondence between the hierarchies of flows on the Hamiltonian variables $\nu$ and $\theta$.

The hierarchy starts at the $k=0$ flow,

$$
h_{\perp}^{(0)}=\nu_{x}, \quad \varpi^{(0)}=\nu, \quad H^{(0)}=\frac{1}{2} \nu^{2}=\frac{1}{2} \theta_{x}^{2},
$$

which produces a convective (traveling wave) equation $\nu_{t}=\nu_{x}$ and $\theta_{t}=\theta_{x}$. Higher flows in the hierarchy are given by

$$
h_{\perp}{ }^{(k)}=\mathcal{R}^{k}\left(\nu_{x}\right), \quad \varpi^{(k)}=\mathcal{R}^{* k}(\nu), \quad k=1,2, \ldots
$$

In particular the $k=+1$ flow is the mKdV equation $\nu_{t}=\nu_{x x x}+\frac{3}{2} \nu^{2} \nu_{x}$ or in potential form $\theta_{t}=\theta_{x x x}+\frac{1}{2} \theta_{x}^{3}$. Each flow (2.16) is bi-Hamiltonian, since $\varpi^{(k)}=\tilde{\mathcal{H}}\left(h_{\perp}{ }^{(k-1)}\right)=$ $\tilde{\mathcal{I}}\left(h_{\perp}{ }^{(k)}\right)$ and hence

$$
-\theta_{t}=\tilde{\mathcal{I}}\left(\delta H^{(k)} / \delta \theta\right)=\tilde{\mathcal{H}}\left(\delta H^{(k-1)} / \delta \theta\right), \quad k=1,2, \ldots,
$$

or equivalently

$$
\nu_{t}=\mathcal{H}\left(\delta H^{(k)} / \delta \nu\right)=\mathcal{I}\left(\delta H^{(k+1)} / \delta \nu\right), \quad k=0,1,2, \ldots
$$

The hierarchy also contains a $k=-1$ flow characterized by the property that it gets mapped into the stationary flow $\theta_{t}=0=\nu_{t}$ under the recursion operators $\mathcal{R}$ and $\tilde{\mathcal{R}}$. Hence in this flow $h_{\perp}{ }^{(-1)}$ and $\varpi^{(-1)}$ satisfy the equation $0=\tilde{\mathcal{H}}\left(h_{\perp}{ }^{(-1)}\right)$ with $\varpi^{(-1)}=\tilde{\mathcal{I}}\left(h_{\perp}{ }^{(-1)}\right)$, producing a nonlocal evolution equation on $\nu$ related in potential form on $\theta$ to the SG equation as shown in more detail later.

Linear combinations of the flows in this hierarchy produce commuting biHamiltonian flows in the non-planar case, $\chi \neq 0$ :

$$
\nu_{t}=h_{\perp}{ }^{(k+1)}+\chi h_{\perp}{ }^{(k)}, \quad \delta H^{(k, \chi)} / \delta \nu=\varpi^{(k+1)}+\chi \varpi^{(k)},
$$

which will be called the $+k$ flow, satisfying the property

$$
\nu_{t}=\mathcal{H}\left(\delta H^{(k, \chi)} / \delta \nu\right)=\mathcal{I}\left(\delta H^{(k+1, \chi)} / \delta \nu\right)
$$

where the Hamiltonians are given by

$$
H=H^{(k, \chi)}:=\chi H^{(k)}+H^{(k+1)} .
$$

In an equivalent potential form, the $+k$ flow is

$$
-\theta_{t}=\varpi^{(k+1)}+\chi \varpi^{(k)}, \quad \delta H^{(k, \chi)} / \delta \theta=h_{\perp}{ }^{(k+1)}+\chi h_{\perp}{ }^{(k)},
$$

which obeys

$$
-\theta_{t}=\tilde{\mathcal{I}}\left(\delta H^{(k, \chi)} / \delta \theta\right)=\tilde{\mathcal{H}}\left(\delta H^{(k-1, \chi)} / \delta \theta\right) .
$$


Independently of $\chi$, associated to the flows on $\nu$ and $\theta$ are a hierarchy of commuting Hamiltonian vector fields $h_{\perp}{ }^{(k)} \partial / \partial \nu$ and a hierarchy of involutive variational covector fields $\varpi^{(k)} d \nu, k=0,1,2, \ldots$, with a corresponding potential form given by switching $\nu$ and $\theta$, as well as $h_{\perp}{ }^{(k)}$ and $\varpi^{(k)}$.

Finally, the motion of $\gamma(t, x)$ determined by these flows will now be derived through the relation $h_{\perp}=h_{\perp}{ }^{(k)}$ producing a hierarchy of non-stretching curve motions from the hierarchy of commuting Hamiltonian vector fields $h_{\perp}{ }^{(k)} \partial / \partial \nu$. The equation of motion for the flow of the curve $\gamma(t, x)$ is given by

$$
\gamma_{t}=h_{\|} X+h_{\perp} J X
$$

with

$$
h_{\|}=D_{x}^{-1}\left(\nu h_{\perp}\right) .
$$

The 0 flow is produced by

$$
h_{\perp}=\nu_{x}, \quad h_{\|}=\frac{1}{2} \nu^{2},
$$

which gives the equation

$$
\gamma_{t}=\frac{1}{2} \nu^{2} X+\nu_{x} J X
$$

Integration by parts and substitution of the Serret-Frenet relations

$$
X=\gamma_{x}, \quad \nu J X=\nabla_{x} \gamma_{x}
$$

then yields

$$
\gamma_{t}=\nabla_{x}^{2} \gamma_{x}+\frac{3}{2}\left|\nabla_{x} \gamma_{x}\right|_{g}^{2} \gamma_{x}, \quad \text { with } \quad\left|\gamma_{x}\right|_{g}=1
$$

This motion will be called a non-stretching $m K d V$ map equation. The higher flows in the hierarchy give analogous higher-order $\mathrm{mKdV}$ map equations, derived recursively via the Serret-Frenet relations and their $x$-derivatives: $\nu_{x} J X=\nabla_{x}(\nu J X)+\nu^{2} X=\nabla_{x}^{2} \gamma_{x}+\nu^{2} \gamma_{x}$ and so on. For instance the +1 flow has

$$
h_{\perp}=\nu_{x x x}+\frac{3}{2} \nu^{2} \nu_{x}, \quad h_{\|}=\nu \nu_{x x}-\frac{1}{2} \nu_{x}^{2}+\frac{3}{8} \nu^{4},
$$

and so

$$
\begin{aligned}
\gamma_{t} & =\left(\nu \nu_{x x}-\frac{1}{2} \nu_{x}^{2}+\frac{3}{8} \nu^{4}\right) X+\left(\nu_{x x x}+\frac{3}{2} \nu^{2} \nu_{x}\right) J X \\
& =\nabla_{x}\left(\frac{3}{2} \nu^{3} J X+\frac{3}{2}\left(\nu^{2}\right)_{x} X+\nabla_{x}^{2}(\nu J X)\right)+\left(\left(\nu^{2}\right)_{x x}-\frac{5}{2} \nu_{x}^{2}+\frac{7}{8} \nu^{4}\right) X
\end{aligned}
$$

which yields

$$
\begin{aligned}
\gamma_{t}=\nabla_{x}^{4} \gamma_{x}+ & \frac{3}{2}\left|\nabla_{x} \gamma_{x}\right|_{g}^{2} \nabla_{x}^{2} \gamma_{x}+3\left(\left|\nabla_{x} \gamma_{x}\right|_{g}^{2}\right)_{x} \gamma_{x} \\
& +\left(\frac{5}{2}\left(\left|\nabla_{x} \gamma_{x}\right|_{g}^{2}\right)_{x x}-\frac{5}{2}\left|\nabla_{x}^{2} \gamma_{x}\right|_{g}^{2}+\frac{27}{8}\left|\nabla_{x} \gamma_{x}\right|_{g}^{4}\right) \gamma_{x}
\end{aligned}
$$

again with $\left|\gamma_{x}\right|_{g}=1$. In general the $+k$ flow for $k=0,1, \ldots$ corresponds to an $\mathrm{mKdV}$ map equation of order $3+2 k$. 
The -1 flow in contrast comes from the equation

$$
0=\varpi=D_{x} h_{\perp}+\nu h_{\|}
$$

which is directly equivalent to $\nabla_{t} X=\varpi J X=0$. This motion is just a wave map equation,

$$
\nabla_{t} \gamma_{x}=0
$$

subject to the non-stretching condition

$$
\left|\gamma_{x}\right|_{g}=1
$$

Furthermore, $t$ can be conformally scaled so that the curve motion has unit speed

$$
\left|\gamma_{t}\right|_{g}=1
$$

In the planar case, i.e. $N=\mathbb{R}^{2}$, the wave map equation reduces to an ordinary wave equation, as the flow on $\nu=-\theta_{x}$ is stationary $\nu_{t}=-\theta_{t x}=0$ due to $\chi=0$. The -1 flow equation for $\chi \neq 0$ is $\nu_{t}=\chi h_{\perp}$ where $\nu=-h_{\|}{ }^{-1} D_{x} h_{\perp}$ and $h_{\|}=\sqrt{1-h_{\perp}{ }^{2}}$, which gives a hyperbolic scalar PDE

$$
\nu_{t x}=-\operatorname{sgn}(\chi) \sqrt{\chi^{2}-\nu_{t}^{2}} \nu
$$

on $\nu$. In potential form, these relations imply $h_{\perp}=\sin \theta, h_{\|}=\cos \theta$, up to a shift in $\theta$ (equivalently, $h_{1}=1, h_{2}=0$ are constants), while $\varpi^{\prime}=D_{x}^{-1} \sin \theta$. Hence the -1 flow in the non-planar case, namely a non-stretching wave map on $N=S^{2}$ or $H^{2}$, is given by

$$
\theta_{t x}=-\chi \sin \theta
$$

which is equivalent to the SG equation.

In addition to all these Hamiltonian flows of curves, there is a trivial flow produced by $h_{\perp}=0, h_{\|}=1$, falling outside the hierarchy. This flow yields the curve motion $\gamma_{t}=X=\gamma_{x}$ which is just a convective (traveling wave) map equation.

In summary: In two-dimensional constant-curvature spaces there is a hierarchy of bi-Hamiltonian commuting flows of non-stretching curves $\gamma(t, x)$; the 0 flow is described by an mKdV map equation, such that the curvature invariant of $\gamma$ in a Frenet frame satisfies the $\mathrm{mKdV}$ equation to within a convective term, while $+k$ flow is a higherorder analog. The wave map equation describes a -1 flow such that it is mapped into the stationary flow under the recursion operator of the hierarchy, with the curvature invariant of $\gamma$ in a covariantly-constant frame satisfying the SG equation.

\section{Frame formulations of integrable flows of curves in three dimensions}

The results just summarized in two dimensions have a natural generalization to three (and higher) dimensions formulated in a more general geometric setting. Let $(N, g)$ be a three-dimensional Riemannian manifold and consider a flow of a curve $\gamma(t, x)$ in $N$. Write $X=\gamma_{x}=q^{a} e_{a}$ for the tangent vector along the curve and $Y=\gamma_{t}=h^{a} e_{a}$ for the evolution vector of the flow of the curve, where $e_{a}$ is any orthonormal frame 
defined in the tangent space $T_{\gamma} N$ on the two-dimensional surface swept out by $\gamma(t, x)$ in $N$. Note the orthonormality of the frame is expressed by $g\left(e_{a}, e_{b}\right)=\delta_{a b}$ (Kronecker symbol); hereafter this frame metric will be used to freely raise and lower frame indices, and the summation convention is assumed on all repeated frame indices. Write $\nabla$ for the covariant derivative determined by the metric $g$ on $N$, and $|X|_{g}^{2}=g(X, X)$ for the metric norm-squared of $X$ on the tangent space $T_{x} N$. Suppose $\gamma(t, x)$ is non-stretching under the flow, so $|X|_{g}^{2}=q^{a} q_{a}=1$ is unit normalized without loss of generality. The frame formulation of covariant derivatives is provided by the introduction of connection 1 -forms $w_{a b}$ (skew in $a b$ ) related to the frame vectors $e_{a}$ through the Cartan structure equations as follows (see also Ref. [13]). On the surface $T_{\gamma} N$ the content of the Cartan equations is that the covariant derivatives $\nabla_{x}$ along the curve and $\nabla_{t}$ along the flow have vanishing torsion

$$
\nabla_{x} \gamma_{t}-\nabla_{t} \gamma_{x}=\left[\gamma_{x}, \gamma_{t}\right]=0
$$

and carry curvature

$$
\left[\nabla_{x}, \nabla_{t}\right]=R\left(\gamma_{x}, \gamma_{t}\right)
$$

given by the Riemann tensor $R(\cdot, \cdot)$ [21] determined from $g$. When expressed in frame components, the torsion and curvature equations look like

$$
0=D_{x} h^{a}-D_{t} q^{a}+h^{b} \Omega_{b}^{a}-q^{b} \Theta_{b}^{a}
$$

and

$$
R\left(\gamma_{x}, \gamma_{t}\right)_{a}^{b}=D_{t} \Omega_{a}^{b}-D_{x} \Theta_{a}^{b}+\Omega_{a}{ }^{c} \Theta_{c}{ }^{b}-\Theta_{a}{ }^{c} \Omega_{c}{ }^{b}
$$

where

$$
\left.\Omega_{a b}=\gamma_{x}\right\lrcorner w_{a b}=g\left(e_{b}, \nabla_{x} e_{a}\right)
$$

and

$$
\left.\Theta_{a b}=\gamma_{t}\right\lrcorner w_{a b}=g\left(e_{b}, \nabla_{t} e_{a}\right)
$$

are the Cartan connection matrices, and where

$$
R\left(\gamma_{x}, \gamma_{t}\right)_{a}{ }^{b}=q^{c} h^{d} R\left(e_{c}, e_{d}\right)_{a}{ }^{b}
$$

is the Cartan curvature matrix. (Alternatively, the connection matrices are seen to arise from the pullback to $T_{\gamma} N$ of the equation [21] $\nabla e_{a}=-w_{a}{ }^{b} \otimes e_{b}$ defining a Riemannian connection $w_{a b}$ in terms of an orthonormal frame $e_{a}$ associated with the Riemannian structure $g$ and $\nabla$ on $N$.)

These frame equations (3.3) to (3.7) will be demonstrated to directly encode pairs of Hamiltonian operators with respect to some Hamiltonian flow variable producing a hierarchy of integrable curve flows if $e_{a}$ and $w_{a b}$ are chosen in a geometrical fashion determined entirely by the curve $\gamma$ and the geometry $(N, g)$, in particular so the frame curvature matrix $R\left(e_{c}, e_{d}\right)_{a}{ }^{b}$ is everywhere constant on $N$.

Constant curvature spaces $(N, g)$ have the distinguishing property that, for all choices of orthonormal frame $e_{a}$,

$$
R\left(e_{c}, e_{d}\right)_{a}^{b}=2 \chi \delta_{a[c} \delta_{d]}^{b} \quad \text { with } \quad \chi=\mathrm{const},
$$


namely such spaces are homogeneous and isotropic, i.e. 3-sphere $N=S^{3}, \chi=1$; 3hyperboloid $N=H^{3}, \chi=-1$; and Euclidean space $N=\mathbb{R}^{3}, \chi=0$. Correspondingly, the local frame structure group is $S O(3)$. Thus, as the frame curvature matrix is constant on $N$, there is a wide freedom available in determining both $e_{a}$ and $w_{a b}$ geometrically in terms of $\gamma$ and $g$ on these spaces. The precise nature of the encoding of Hamiltonian operators in the frame structure equations depends essentially on whether the frame $e_{a}$ is adapted to the tangent vector $X$ of the curve.

\subsection{Parallel frames and vector $m K d V$ Hamiltonian operators}

To begin, for the situation of an adapted moving frame $e_{a}$, the components of $X$ are given by constants $q^{a}$. The Hamiltonian flow variable in this case will be the frame components $v^{a}$ of $v=\nabla_{x} X$, with the associated Hamiltonian vector and covector field variables ${h_{\perp}}^{a}, \varpi^{a}$ given by the frame components of $Y_{\perp}=Y-g(Y, X) X, \varpi=\nabla_{t} X$. The flow equation of motion on $v$ comes from the part of the curvature structure equation (3.2) projected along the tangent direction of $\gamma, \nabla_{t} v=\nabla_{x} \varpi+\chi Y_{\perp}$ which makes use of the constant curvature property of $(N, g)$. The remaining part of the curvature structure equation (projected into the normal space of $\gamma$ ) combined with the torsion structure equation (3.1) allow $\nabla_{t}, \nabla_{x}, Y_{\|}=g(Y, X)$ to be expressed in terms of $\varpi, Y_{\perp}$. These expressions look simplest if the connection $w_{a b}$ is also adapted to the curve by putting $\left(\nabla_{x}\left(e_{a}\right)_{\perp}\right)_{\perp}=0$, in which case $e_{a}$ becomes precisely a parallel frame [10] such that $\nabla_{x}\left(e_{a}\right)_{\perp}=-v_{a} X$. In detail: $\Omega_{a b}=2 q_{[a} v_{b]}$ is the connection equation of the parallel frame; the torsion equation yields

$$
\varpi^{a}=h_{\|} v^{a}+D_{x} h_{\perp}^{a}, \quad D_{x} h_{\|}=h_{\perp}{ }^{a} v_{a}
$$

where $h_{\|}=h^{a} q_{a}$, and the normal part of the curvature equation yields

$$
D_{x} \Theta_{a b}=2 \varpi_{[a} v_{b]}+2 q_{[a} D_{x} \varpi_{b]} .
$$

The tangential part of the curvature equation then gives

$$
v_{t}^{a}=D_{x} \varpi^{a}+2 v_{b} D_{x}^{-1}\left(\varpi^{[a} v^{b]}\right)+\chi h_{\perp}^{a} .
$$

Because of the non-stretching condition on $X$, observe $v^{a} q_{a}=\varpi^{a} q_{a}=0$ and likewise $h_{\perp}{ }^{a} q_{a}=0$. It is now convenient to regard $v^{a}, \varpi^{a},{h_{\perp}}^{a}$ as 2-component vectors $\boldsymbol{v}, \varpi, \boldsymbol{h}_{\perp}$ and employ an index-free vector notation for writing out the Hamiltonian operators. Hence, the flow equation is given by

$$
\boldsymbol{v}_{t}=\mathcal{H}(\varpi)+\chi \mathcal{J}^{-1}(\varpi)=\mathcal{R}\left(\boldsymbol{h}_{\perp}\right)+\chi \boldsymbol{h}_{\perp}
$$

in terms of the operators

$$
\mathcal{H}=D_{x}+* \boldsymbol{v} D_{x}^{-1}(* \boldsymbol{v} \cdot), \quad \mathcal{J}=D_{x}+\boldsymbol{v} D_{x}^{-1}(\boldsymbol{v} \cdot), \quad \mathcal{R}=\mathcal{H} \circ \mathcal{J},
$$

where $*$ is the Hodge-star operator on vectors in two dimensions obeying the product identity $\ddagger \boldsymbol{C}\lrcorner(\boldsymbol{B} \wedge \boldsymbol{A})=(* \boldsymbol{A} \cdot \boldsymbol{B}) * \boldsymbol{C}$. As proved in Ref. 9] using the framework

$\ddagger$ Throughout, in any dimension, $\lrcorner$ denotes the interior product between a vector and a 1 -form or 2 -form, as well as the contraction of a vector against an antisymmetric tensor; $\wedge$ denotes the exterior 
developed in Ref. [20, 19], $\mathcal{H}$ and $\mathcal{J}$ are a pair of cosymplectic and symplectic Hamiltonian operators, and the formal inverse $\mathcal{J}^{-1}=\mathcal{I}$ as defined on the $x$-jet space of $\boldsymbol{v}(t, x)$ is a cosymplectic operator compatible with $\mathcal{H}$. Moreover, $\mathcal{R}$ is a hereditary recursion operator for a hierarchy of Hamiltonians flows that are each invariant under the frame structure group. Group-invariance requires the flow components to be a covariant vector function $\boldsymbol{h}_{\perp}=\boldsymbol{h}_{\perp}\left(\boldsymbol{v}, \boldsymbol{v}_{x}, \boldsymbol{v}_{x x}, \ldots\right)$ on the jet space, so accordingly the flow equation has an $O(2)$-invariant vector form, where this group $O(2)$ is the isotropy subgroup of the frame structure group leaving invariant $X$ for an adapted (parallel) frame. Because a parallel frame is determined geometrically (by the curve $\gamma$ ) up to a rigid $S O(2)$ rotation, the Hamiltonian variable $\boldsymbol{v}$ represents differential covariants of $\gamma$ [22].

The 0 flow in the hierarchy is produced by $\boldsymbol{h}_{\perp}=\boldsymbol{v}_{x}$, giving the vector PDE

$$
\boldsymbol{v}_{t}=\boldsymbol{v}_{x x x}+\frac{3}{2}|\boldsymbol{v}|^{2} \boldsymbol{v}_{x}+\chi \boldsymbol{v}_{x}
$$

This flow is a well-known vector generalization [23] of the mKdV equation, to within a convective term that can be absorbed by a Galilean transformation $x \rightarrow x-\chi t$, $t \rightarrow t$. The $+k$ flow as obtained from $\boldsymbol{h}_{\perp}=\mathcal{R}^{k}\left(\boldsymbol{v}_{x}\right)$ gives a vector mKdV equation of higher-order $3+2 k$ on $\boldsymbol{v}$. These flows correspond to geometric motions of the curve $\gamma$,

$$
\gamma_{t}=f\left(\gamma_{x}, \nabla_{x} \gamma_{x}, \nabla_{x}^{2} \gamma_{x}, \ldots\right)=h^{a} e_{a}
$$

subject to the non-stretching condition $\left|\gamma_{x}\right|_{g}=1$. The equation of motion is obtained from the decompositions $e_{a}=q_{a} X+\left(e_{a}\right)_{\perp}$ and $h^{a}=h_{\|} q^{a}+h_{\perp}{ }^{a}$ with $h_{\|}=D_{x}^{-1}\left(h_{\perp}{ }^{a} v_{a}\right)$, after substitution of the expressions $h_{\perp}{ }^{a}=h_{\perp}{ }^{a}\left(v^{b}, v_{x}^{b}, v_{x x}^{b}, \ldots\right)$ followed by use of the relations

$$
\begin{aligned}
v^{a}\left(e_{a}\right)_{\perp}=\nabla_{x} \gamma_{x}, \quad v_{x}^{a}\left(e_{a}\right)_{\perp} & =\nabla_{x}\left(v^{a} e_{a}\right)-v^{a} \nabla_{x} e_{a} \\
& =\nabla_{x}^{2} \gamma_{x}+\left|\nabla_{x} \gamma_{x}\right|_{g}^{2} \gamma_{x},
\end{aligned}
$$

and so on, where

$$
\nabla_{x}\left(e_{a}\right)_{\perp}=-v_{a} \gamma_{x}
$$

In particular, for the 0 flow, $h_{\perp}{ }^{a}=v_{x}^{a}, h_{\|}=\frac{1}{2} v^{a} v_{a}$, thus

$$
\gamma_{t}=h^{a} e_{a}=\frac{1}{2} v^{a} v_{a} X+v_{x}^{a}\left(e_{a}\right)_{\perp}=\nabla_{x}^{2} \gamma_{x}+\frac{3}{2}\left|\nabla_{x} \gamma_{x}\right|_{g}^{2} \gamma_{x}
$$

Note the trivial flow given by $\boldsymbol{h}_{\perp}=0, h_{\|}=1$ corresponds to the motion $\gamma_{t}=\gamma_{x}$ which is a convective (traveling wave) map equation.

There is also a -1 flow contained in the hierarchy, with the property that $\boldsymbol{h}_{\perp}$ is annihilated by the symplectic operator $\mathcal{J}$ and hence gets mapped into $\boldsymbol{h}_{\perp}=0$ under product taking a pair of vectors into an antisymmetric tensor, as well as the ordinary wedge product between forms; the Hodge-star operator $*$ is mapping between a vector and a rank $n-1$ skew tensor in $n>2$ dimensions or between vectors in the $n=2$ dimensional case, with $*^{2}=-1$. In addition, vectors and 1-forms will be identified using the frame metric. 
the recursion operator $\mathcal{R}$. Geometrically this flow means simply $\mathcal{J}\left(\boldsymbol{h}_{\perp}\right)=\varpi=0$ so $\gamma$ satisfies the equation of motion

$$
\varpi=\nabla_{t} \gamma_{x}=0
$$

which is the wave map equation on the target space $N=S^{3}, H^{3}, \mathbb{R}^{3}$. The resulting vector $\mathrm{PDE}$ is a nonlocal evolution equation

$$
\boldsymbol{v}_{t}=\chi \boldsymbol{h}_{\perp}
$$

where $\boldsymbol{h}_{\perp}$ satisfies

$$
0=\varpi=D_{x} \boldsymbol{h}_{\perp}+h_{\|} \boldsymbol{v} \quad \text { and } \quad D_{x} h_{\|}=\boldsymbol{h}_{\perp} \cdot \boldsymbol{v} .
$$

Note this PDE possesses the conservation law $0=D_{x}\left(h_{\|}^{2}+\left|\boldsymbol{h}_{\perp}\right|^{2}\right)=\nabla_{x}\left|\gamma_{t}\right|_{g}^{2}$ and so a conformal scaling of $t$ can be used to put

$$
1=\left|\gamma_{t}\right|_{g}^{2}=h_{\|}^{2}+\left|\boldsymbol{h}_{\perp}\right|^{2} .
$$

This relation enables $h_{\|}, \boldsymbol{h}_{\perp}$ to be expressed entirely in terms of $\boldsymbol{v}$. Its geometrical meaning is that the flow is conformally equivalent to one with uniform speed. Consequently, the -1 flow vector PDE reduces to

$$
\boldsymbol{v}_{t}=-\operatorname{sgn}(\chi) D_{x}^{-1}\left(\sqrt{\chi^{2}-\left|\boldsymbol{v}_{t}\right|^{2}} \boldsymbol{v}\right)
$$

which is equivalent to a hyperbolic vector equation

$$
\boldsymbol{v}_{t x}=-\sqrt{1-\left|\boldsymbol{v}_{t}\right|^{2}} \boldsymbol{v}
$$

after a further scaling $t \rightarrow \chi^{-1} t$. This PDE is related to [24] a vector SG equation known from $O(4)$ sigma models, which will be derived later in potential form.

Theorem 1: In three dimensions there is a hierarchy of group-invariant integrable flows of curves $\gamma(t, x)$ in any constant-curvature space $(N, g) \simeq S^{3}, H^{3}, \mathbb{R}^{3}$. The 0 flow is a non-stretching mKdV map equation

$$
\gamma_{t}=\nabla_{x}^{2} \gamma_{x}+\frac{3}{2}\left|\nabla_{x} \gamma_{x}\right|_{g}^{2} \gamma_{x}, \quad\left|\gamma_{x}\right|_{g}=1,
$$

while the $+k$ flow is a higher-order analog $\gamma_{t}=f\left(\gamma_{x}, \nabla_{x} \gamma_{x}, \ldots, \nabla_{x}^{2 k+2} \gamma_{x}\right)$. The -1 flow is a non-stretching wave map equation

$$
\nabla_{t} \gamma_{x}=0, \quad\left|\gamma_{x}\right|_{g}=\left|\gamma_{t}\right|_{g}=1
$$

which is equivalent to the $O(4)$ sigma model.

Group-invariance has the geometric meaning here of covariance of the equation of motion (3.15) of $\gamma(t, x)$ under the group of isometries of $(N, g)$, as implied by the $O(2)$ invariance property of the vector expressions $\boldsymbol{h}_{\perp}=\boldsymbol{h}_{\perp}\left(\boldsymbol{v}, \boldsymbol{v}_{x}, \boldsymbol{v}_{x x}, \ldots\right)$ on the $x$-jet space of $\boldsymbol{v}(t, x)$. Note these expressions for each $+k$ flow, $k=0,1, \ldots$, are explicit polynomials, while the -1 flow is only given by an implicit nonlocal expression in this setting.

Similarly to the derivation in two dimensions, associated to the curve flows in three dimensions is a hierarchy of commuting Hamiltonian vector fields $\boldsymbol{h}_{\perp}{ }^{(k)} \cdot \partial / \partial \boldsymbol{v}$ and involutive variational covector fields $\varpi^{(k)} \cdot d \boldsymbol{v}, k=0,1,2, \ldots$, generated by the 
recursion operator $\mathcal{R}$ and its adjoint $\mathcal{R}^{*}$. The hierarchy starts from $\boldsymbol{h}_{\perp}{ }^{(0)}=\boldsymbol{v}_{x}$, $\varpi^{(0)}=\boldsymbol{v}$, which has the geometrical meaning that the Hamiltonian vector field $\boldsymbol{h}_{\perp}{ }^{(0)} \cdot \partial / \partial \boldsymbol{v}$ is the infinitesimal generator of $x$-translations, where $x$ is the arclength along the curve $\gamma$. This hierarchy has a natural bi-Hamiltonian structure $\boldsymbol{h}_{\perp}{ }^{(k)}=$ $\mathcal{H}\left(\delta H^{(k)} / \delta \boldsymbol{v}\right)=\mathcal{I}\left(\delta H^{(k+1)} / \delta \boldsymbol{v}\right)$ with $\varpi^{(k)}=\delta H^{(k)} / \delta \boldsymbol{v}$ determining the Hamiltonians $H=H\left(\boldsymbol{v}, \boldsymbol{v}_{x}, \boldsymbol{v}_{x x}, \ldots\right)$. Consequently, the curve flows themselves are bi-Hamiltonian:

$$
\boldsymbol{v}_{t}=\mathcal{H}\left(\delta H^{(k, \chi)} / \delta \boldsymbol{v}\right)=\mathcal{I}\left(\delta H^{(k+1, \chi)} / \delta \boldsymbol{v}\right)
$$

where

$$
H^{(k, \chi)}=\chi H^{(k)}+H^{(k+1)} .
$$

\subsection{Covariantly-constant frames and vector potential $m K d V$ Hamiltonian operators}

The previous results will now be extended to the situation of a non-adapted moving frame $e_{a}$ for curve flows in the same constant-curvature spaces. In this case the frame components $q^{a}$ of the tangent vector $X$ are (non-constant) functions of $t, x$, and as a consequence the most natural Hamiltonian variable will be given by $v=X$ itself. Compared to the previous case when $v=\nabla_{x} X$, the roles of the associated variables $Y_{\perp}=Y-Y_{\|} X, \varpi=\nabla_{t} X$ are switched here to be Hamiltonian covector and vector field variables, respectively. This relationship is analogous to going to a potential formulation in the Hamiltonian setting, similarly to the situation in two dimensions. The flow equation of motion on $v$ now comes from the part of the torsion structure equation (3.1) projected into the normal space of $\gamma, \nabla_{t} X=\left(\nabla_{x} Y\right)_{\perp}=\nabla_{x} Y_{\perp}+g\left(Y_{\perp}, \nabla_{x} X\right) X+Y_{\|} \nabla_{x} X$, while the curvature structure equation (3.2) and the tangential part of the torsion structure equation are used to express $\nabla_{t}, \nabla_{x}, Y_{\|}$in terms of $\varpi, Y_{\perp}$.

There is a quite simple encoding of Hamiltonian operators in the frame components of these equations if the frame $e_{a}$ is chosen to be covariantly-constant $\nabla_{x} e_{a}=0$, i.e. parallel transported, along the curve. (In a geometrical sense, whereas a parallel frame is completely adapted to the curve, a covariantly-constant frame is minimally adapted yet still determined entirely by geometrical considerations.) To give some details: the connection equation is simply $\Omega_{a b}=0$ while the curvature equation reduces to $D_{x} \Theta_{a b}=-2 \chi q_{[a} h_{b]}$. The torsion equation yields $D_{x} h_{\|}=h_{\perp_{a}} q_{x}^{a}$ and so the flow equation becomes

$$
q_{t}^{a}=\varpi^{a}-2 \chi q_{b} D_{x}^{-1}\left(q^{[a} h_{\perp}^{b]}\right)
$$

with

$$
\varpi^{a}=D_{x} h_{\perp}^{a}+h_{\|} q_{x}^{a}+q^{a} h_{\perp b} q_{x}^{b} .
$$

Note the non-stretching condition on $X$ implies $\varpi^{a} q_{a}=h_{\perp}{ }^{a} q_{a}=0$ as before, but now $q^{a}$ is the Hamiltonian variable and obeys the constraint $q^{a} q_{a}=1$. For ease of notation, $q^{a}, \varpi^{a}, h_{\perp}{ }^{a}$ will be written as 3 -component vectors with $\vec{\varpi}, \vec{h}_{\perp} \perp \vec{q}$, subject to $|\vec{q}|=1$. 
A useful vector product identity $甘$ is $\vec{C}\lrcorner(\vec{B} \wedge \vec{A})=\vec{C} \times(\vec{B} \times \vec{A})$ in three dimensions. This leads to the operators

$$
\mathcal{I}=-\vec{q} \times D_{x}^{-1}(\vec{q} \times)
$$

and

$$
\mathcal{H}=D_{x}+\vec{q}_{x} D_{x}^{-1}\left(\vec{q}_{x} \cdot\right)+\vec{q}\left(\vec{q}_{x} \cdot\right)
$$

in terms of which the flow equation is given by

$$
\vec{q}_{t}=\chi \mathcal{I}\left(\vec{h}_{\perp}\right)+\mathcal{H}\left(\vec{h}_{\perp}\right) .
$$

The following result can be proved by computations similar to Ref. [9] based on the framework presented in Ref. [20, 19]. Details will be given elsewhere.

Theorem 2: $\mathcal{I}, \mathcal{H}$ are a pair of compatible Hamiltonian (cosymplectic) operators and obey $\vec{q} \cdot \mathcal{I}=\vec{q} \cdot \mathcal{H}=0$, with respect to the constrained Hamiltonian variable $\vec{q}(t, x)$. The inverse of $\mathcal{I}$ is a Hamiltonian symplectic operator

$$
\mathcal{J}=\mathcal{I}^{-1}=-\vec{q} \times D_{x}\left((\vec{q} \times)-\vec{q} D_{x}^{-1}\left(\left(\vec{q} \times \vec{q}_{x}\right) \cdot\right)\right)
$$

whose domain is defined on the $x$-jet space coordinates $\perp \vec{q}$. $甘$ Then the flow equation on $\vec{q}$ takes the form $\vec{q}_{t}=\chi \vec{\varpi}^{\prime}+\mathcal{R}\left(\vec{\varpi}^{\prime}\right)$ in terms of the operator $\mathcal{R}=\mathcal{H} \circ \mathcal{J}$, where $\vec{\varpi}^{\prime}=\mathcal{I}\left(\vec{h}_{\perp}\right)$ is the inverse image of $\vec{\varpi}=\mathcal{H}\left(\vec{h}_{\perp}\right)$ under $\mathcal{R}$.

As a result, $\mathcal{R}=\mathcal{H} \circ \mathcal{J}$ will be a hereditary recursion operator for commuting Hamiltonian vector fields $\vec{\varpi}^{(k)} \cdot \partial / \partial \vec{q}, k=0,1,2, \ldots$, starting from $\vec{\varpi}^{(0)}=\vec{q}_{x}$, the infinitesimal generator of $x$-translations in terms of the arclength $x$ along the curve $\gamma$. Likewise the adjoint operator $\mathcal{R}^{*}=\mathcal{J} \circ \mathcal{H}$ will be a hereditary recursion operator for involutive variational covector fields $\vec{h}_{\perp}{ }^{(k)} \cdot d \vec{q}, k=0,1,2, \ldots$, related by $\vec{h}_{\perp}{ }^{(k)}=\mathcal{J}\left(\vec{\varpi}^{(k)}\right)$ and thereby starting from $\vec{h}_{\perp}{ }^{(0)}=\mathcal{J}\left(\vec{q}_{x}\right)=\vec{q}_{x x}+\left|\vec{q}_{x}\right|^{2} \vec{q}$. This hierarchy has a natural bi-Hamiltonian structure $\vec{\varpi}^{(k+1)}=\mathcal{I}\left(\vec{h}_{\perp}{ }^{(k+1)}\right)=\mathcal{H}\left(\vec{h}_{\perp}{ }^{(k)}\right)$ with $\vec{h}_{\perp}{ }^{(k)}=-\delta H^{(k)} / \delta \vec{q}$ determining the Hamiltonians $H=H\left(\vec{q}, \vec{q}_{x}, \vec{q}_{x x}, \ldots\right)$. Because of the constraint $|\vec{q}|=1$, the variational derivatives involve a Lagrangian multiplier term $\lambda(|\vec{q}|-1)$ in the Hamiltonians to enforce the constraint condition $\vec{q} \cdot \delta H / \delta \vec{q}=0$. For instance, $\vec{h}_{\perp}{ }^{(0)}=\vec{q}_{x x}+\left|\vec{q}_{x}\right|^{2} \vec{q}=-\delta H^{(0)} / \delta \vec{q}$ determines $H^{(0)}=\frac{1}{2}\left|\vec{q}_{x}\right|^{2}+\lambda(|\vec{q}|-1)$ where, after variational derivatives are evaluated, $\lambda=-\left|\vec{q}_{x}\right|^{2}$.

The hierarchy $\vec{\varpi}^{\prime}=\vec{\varpi}^{(k)}, k=0,1,2, \ldots$, produces commuting bi-Hamiltonian flows on $\vec{q}$ given by constrained vector PDEs

$$
\vec{q}_{t}=\chi \vec{\varpi}^{(k)}+\vec{\varpi}^{(k+1)}, \quad|\vec{q}|=1 .
$$

Their Hamiltonian structure looks like

$$
-\vec{q}_{t}=\mathcal{I}\left(\delta H^{(k, \chi)} / \delta \vec{q}\right)=\mathcal{H}\left(\delta H^{(k-1, \chi)} / \delta \vec{q}\right)
$$

$\ddagger$ Throughout, $\times$ denotes the standard cross-product on three-dimensional vectors.

$\ddagger$ Namely, the coordinate space $\left\{\left(\vec{q}_{x}, \vec{q}_{x x}, \ldots\right)_{\perp}=\left(\vec{q}_{x}, \vec{q}_{x x}+\left|\vec{q}_{x}\right|^{2} \vec{q}, \ldots\right)\right\}$ consisting of those vectors $\perp$ $\vec{q}$ derived from the differential consequences of $|\vec{q}|^{2}=1$. 
where

$$
H^{(k, \chi)}=\chi H^{(k)}+H^{(k+1)}
$$

are the Hamiltonians as before. These PDEs have an $O(3)$-invariant form on the $x$ jet space of $\vec{q}(t, x)$, where $\vec{\varpi}^{\prime}=\vec{\varpi}^{(k)}\left(\vec{q}, \vec{q}_{x}, \vec{q}_{x x}, \ldots\right)$ and $\vec{h}_{\perp}=\vec{h}_{\perp}{ }^{(k)}\left(\vec{q}, \vec{q}_{x}, \vec{q}_{x x}, \ldots\right)$ are covariant vector functions given by explicit polynomial expressions. Correspondingly, the curve $\gamma(t, x)$ undergoes a group-invariant non-stretching motion

$$
\gamma_{t}=f\left(\gamma_{x}, \nabla_{x} \gamma_{x}, \nabla_{x}^{2} \gamma_{x}, \ldots\right)
$$

which is related to these vector expressions by substitution of

$$
q^{a} e_{a}=\gamma_{x}, \quad q_{x}^{a} e_{a}=\nabla_{x}\left(q^{a} e_{a}\right)=\nabla_{x} \gamma_{x}, \quad q_{x x}^{a} e_{a}=\nabla_{x}\left(q_{x}^{a} e_{a}\right)=\nabla_{x}^{2} \gamma_{x}
$$

and so on, into the flow equation given by $f=h^{a} e_{a}$ with $h^{a}=h_{\|} q^{a}+h_{\perp}{ }^{a}$ and $h_{\|}=D_{x}^{-1}\left(h_{\perp_{a}} q_{x}^{a}\right)$.

The 0 flow on $\vec{q}$ corresponds to the curve motion specified by the non-stretching mKdV map equation (3.25), which yields

$$
\vec{q}_{t}=\vec{q}_{x x x}+\frac{3}{2}\left(\left|\vec{q}_{x}\right|^{2} \vec{q}\right)_{x}+\chi \vec{q}_{x} .
$$

This flow has the form of a vector $\mathrm{mKdV}$ potential equation subject to the constraint $|\vec{q}|=1$. More generally, the $+k$ flow in the hierarchy is a higher-order mKdV constrained potential equation arising from the mKdV map equation of order $3+2 k$ in theorem 2 through the geometrical relation between the frame components $\vec{h}_{\perp}$ of the curve motion $\left(\gamma_{t}\right)_{\perp}$ in the covariantly-constant frame and the parallel frame. This relation is expressed via the correspondence

$$
\begin{aligned}
& \boldsymbol{v} \leftrightarrow \nabla_{x} \gamma_{x} \leftrightarrow \vec{q}_{x}, \\
& \boldsymbol{v}_{x} \leftrightarrow\left(\nabla_{x}^{2} \gamma_{x}\right)_{\perp} \leftrightarrow D_{x}^{\perp} \vec{q}_{x},
\end{aligned}
$$

and so on, where (on the domain of $x$-jet space coordinates $\perp \vec{q}$ )

$$
D_{x}^{\perp}=D_{x}-\vec{q} \vec{q} \cdot D_{x}=D_{x}+\vec{q}\left(\vec{q}_{x} \cdot\right) .
$$

Under this correspondence, the Hamiltonian operator (3.32) and symplectic operator (3.34) in the covariantly-constant frame carry over respectively to the symplectic and cosymplectic operators (3.13) in the parallel frame.

Proposition 3: For any mKdV flow $\boldsymbol{v}_{t}=\boldsymbol{h}_{\perp}{ }^{(\chi)}\left(\boldsymbol{v}, \boldsymbol{v}_{x}, \boldsymbol{v}_{x x}, \ldots\right)$ in the parallel frame (where $\boldsymbol{h}_{\perp}{ }^{(\chi)}$ denotes a linear combination of Hamiltonian vector fields $\boldsymbol{h}_{\perp}{ }^{(k+1)}+\chi \boldsymbol{h}_{\perp}{ }^{(k)}$, $k=0,1, \ldots)$, the corresponding constrained potential mKdV flow in the covariantlyconstant frame is explicitly given by $\vec{q}_{t}=-\vec{q} \times D_{x}^{-1}\left(\vec{q} \times \vec{h}_{\perp}{ }^{(\chi)}\left(\vec{q}_{x}, D_{x}^{\perp} \vec{q}_{x},\left(D_{x}^{\perp}\right)^{2} \vec{q}_{x}, \ldots\right)\right)$. This correspondence extends to the convective (traveling wave) flow in the two frames.

The converse correspondence is expressed via the augmented relations

$$
\begin{aligned}
& \vec{q} \leftrightarrow \gamma_{x} \leftrightarrow(\mathbf{0}, 1) \\
& \vec{q}_{x} \leftrightarrow \nabla_{x} \gamma_{x} \leftrightarrow(\boldsymbol{v}, 0), \\
& \vec{q}_{x x} \leftrightarrow \nabla_{x}^{2} \gamma_{x} \leftrightarrow \boldsymbol{D}_{x}(\boldsymbol{v}, 0)
\end{aligned}
$$


and so on, using the derivative operator

$$
\boldsymbol{D}_{x}=D_{x}+(* \boldsymbol{v}, 0) \times .
$$

(A helpful identity relating 2-component and 3-component vectors here is $(\boldsymbol{v}, 0) \times(\mathbf{0}, 1)=$ $(* \boldsymbol{v}, 0)$.) This operator comes from the geometrical form of the parallel frame $e_{a}$ in three dimensions as follows: Write $\left(e_{a}\right)_{\perp} \leftrightarrow \boldsymbol{e}_{\perp}$ so $e_{a}=\left(\boldsymbol{e}_{\perp}, X\right)$ is adapted to $X=\gamma_{x}$. Then the conditions $\nabla_{x}\left(e_{a}\right)_{\perp}=-v_{a}$ and $\nabla_{x} X=v$ for $e_{a}$ to be parallel [10] are expressed by

$$
\nabla_{x}\left(\boldsymbol{e}_{\perp}, X\right)=(-\boldsymbol{v} \otimes X, v)=-(* \boldsymbol{v}, 0) \times\left(\boldsymbol{e}_{\perp}, X\right)
$$

where

$$
g\left(v, \boldsymbol{e}_{\perp}\right)=\boldsymbol{v}, \quad g\left(X, \boldsymbol{e}_{\perp}\right)=g(X, v)=0,
$$

with $\boldsymbol{v} \leftrightarrow v_{a}$. Hence a parallel frame has the invariant characterization $\boldsymbol{\nabla}_{x} e_{a}=0$ where $\boldsymbol{\nabla}_{x}$ is the covariant version of $\boldsymbol{D}_{x}$.

Proposition 4: Any constrained potential mKdV flow $\vec{q}_{t}=\vec{\varpi}^{(\chi)}\left(\vec{q}, \vec{q}_{x}, \vec{q}_{x x}, \ldots\right)$ in the covariantly-constant frame (where $\vec{\varpi}^{(\chi)}$ denotes a linear combination of Hamiltonian vector fields $\left.\vec{\varpi}^{(k+1)}+\chi \vec{\varpi}^{(k)}, \quad k=0,1, \ldots\right)$ has the corresponding form $\boldsymbol{v}_{t}=$ $\tilde{\mathcal{H}}\left(\varpi^{(\chi)}\left((\mathbf{0}, 1),(\boldsymbol{v}, 0), \boldsymbol{D}_{x}(\boldsymbol{v}, 0), \ldots\right)\right)$ given by an mKdV flow, using the Hamiltonian operator $\tilde{\mathcal{H}}=D_{x}+* \boldsymbol{v} D_{x}^{-1}(* \boldsymbol{v} \cdot)$ from the parallel frame.

The constraint $|\vec{q}|=1$ can be resolved by the introduction of variables

$$
\vec{q}=(k, \boldsymbol{k}),
$$

defined relative to any fixed unit vector $\hat{n}$ by $k=\vec{q} \cdot \hat{n}$ and $\boldsymbol{k}=\vec{q}-k \hat{n}$, satisfying

$$
1=(k)^{2}+|\boldsymbol{k}|^{2} .
$$

Elimination of the scalar variable $k$ then leads to unconstrained potential $\mathrm{mKdV}$ flows in terms of the 2 -component vector variable $\boldsymbol{k}$. To within a convective term, the 0 flow is given by

$$
\boldsymbol{k}_{t}=\boldsymbol{k}_{x x x}+\frac{3}{2}\left(\left(|\boldsymbol{k}|^{2}\left(1-|\boldsymbol{k}|^{2}\right)^{-1}\left(|\boldsymbol{k}|_{x}\right)^{2}+\left|\boldsymbol{k}_{x}\right|^{2}\right) \boldsymbol{k}\right)_{x}
$$

which is a vector generalization of the potential $\mathrm{mKdV}$ equation. In particular, for $\vec{q}=(\cos \theta, \sin \theta, 0)$ lying in a plane, where $k=\cos \theta, \boldsymbol{k}=(\sin \theta, 0)$, then $\theta$ satisfies the potential $\mathrm{mKdV}$ equation $\theta_{t}=\theta_{x x x}+\frac{1}{2} \theta_{x}^{3}$. This reduction has the geometrical meaning that the curve $\gamma(t, x)$ is restricted to lie on a totally-geodesic two dimensional subspace given by the sphere $S^{2}$ when $\chi=1$ or the hyperboloid $H^{2}$ when $\chi=-1$ or the plane $\mathbb{R}^{2}$ when $\chi=0$ in the respective constant-curvature spaces $N=S^{3}, H^{3}, \mathbb{R}^{3}$.

The same correspondence also applies to the curve motion specified by the nonstretching wave map equation (3.26), with $t$ conformally scaled so that $1=\left|\gamma_{t}\right|_{g}$, which gives the -1 flow in the hierarchy. This flow is characterized by the kernel of the recursion operator $0=\vec{\varpi}=\mathcal{R}\left(\vec{\varpi}^{\prime}\right)=\mathcal{H}\left(\vec{h}_{\perp}\right)$ in the covariantly-constant frame, corresponding to the kernel of the symplectic operator in the parallel frame. Hence the -1 flow equation is given by $\vec{q}_{t}=\chi \vec{\varpi}^{\prime}=\chi \mathcal{I}\left(\vec{h}_{\perp}\right)$ with $\vec{h}_{\perp}$ determined by the geometrical equation $\vec{\varpi}=D_{x} \vec{h}=0$ where the vector $\vec{h}=h_{\|} \vec{q}+\vec{h}_{\perp}$ is thus constant. This vector 
also satisfies $|\vec{h}|=\left|\gamma_{t}\right|_{g}=1$, which allows putting $k=h_{\|}, \boldsymbol{k}=\vec{q}-h_{\|} \vec{h}$, relative to $\vec{h}=\hat{n}$. Then the tangential and normal parts of the -1 flow equation on $\vec{q}$ yield nonlocal evolution equations $\boldsymbol{k}_{t}=-\chi k D_{x}^{-1} \boldsymbol{k}$ and $k_{t}=\chi \boldsymbol{k} \cdot D_{x}^{-1} \boldsymbol{k}$, with the identity $1=(k)^{2}+|\boldsymbol{k}|^{2}$. Thus, after the scaling $t \rightarrow \chi^{-1} t$ is made, the 2-component vector variable $\boldsymbol{k}$ satisfies the hyperbolic PDE

$$
\left(\sqrt{\left(1-|\boldsymbol{k}|^{2}\right)^{-1}} \boldsymbol{k}_{t}\right)_{x}=-\boldsymbol{k}
$$

which is a well-known vector generalization [25, 26] of the SG equation. In particular, under the reduction $k=\cos \theta, \boldsymbol{k}=(\sin \theta, 0)$, considered earlier where the curve $\gamma(t, x)$ lies on a totally-geodesic two dimensional subspace in $N$, the variable $\theta$ satisfies the ordinary SG equation.

The bi-Hamiltonian operators in theorem 2 for the hierarchy of potential $\mathrm{mKdV}$ flows are readily formulated by using the unconstrained variable $\boldsymbol{k}$, providing a direct 2-component vector generalization of the well-known bi-Hamiltonian structure of the SG hierarchy. Write $\boldsymbol{h}^{\perp}=\vec{h}_{\perp}-\hat{n} \cdot \vec{h}_{\perp} \hat{n}, \varpi^{\perp}=\vec{\varpi}-\hat{n} \cdot \vec{\varpi} \hat{n}$. Then we have

$$
\boldsymbol{k}_{t}=\chi \mathcal{I}^{\perp}\left(\boldsymbol{h}^{\perp}\right)+\mathcal{H}^{\perp}\left(\boldsymbol{h}^{\perp}\right)
$$

where the operators

$$
\mathcal{I}^{\perp}=k D_{x}^{-1}\left(k+\boldsymbol{k} k^{-1} \boldsymbol{k} \cdot\right)-* \boldsymbol{k} D_{x}^{-1}(* \boldsymbol{k} \cdot)
$$

and

$$
\mathcal{H}^{\perp}=D_{x}+D_{x}\left(\boldsymbol{k} D_{x}^{-1}\left(\left(\boldsymbol{k}_{x} \cdot\right)-k^{-1} k_{x} \boldsymbol{k} \cdot\right)\right)
$$

are given by the normal parts of $\mathcal{I}, \mathcal{H}$ relative to $\hat{n}$. The normal part of $\mathcal{J}$ relative to $\hat{n}$ yields an inverse operator for $\mathcal{I}^{\perp}$,

$$
\begin{aligned}
\mathcal{J}^{\perp}= & D_{x}+\boldsymbol{k}\left(\left(\boldsymbol{k}_{x} \cdot\right)-k^{-1} k_{x} \boldsymbol{k} \cdot\right) \\
& -k * \boldsymbol{k} D_{x}^{-1}\left(|\boldsymbol{k}|\left(k|\boldsymbol{k}|^{-1}\right)_{x}(* \boldsymbol{k} \cdot)+\left(\boldsymbol{k}_{x} \cdot * \boldsymbol{k}\right) k^{-1}|\boldsymbol{k}|^{-2} \boldsymbol{k} \cdot\right) .
\end{aligned}
$$

With respect to the unconstrained Hamiltonian variable $\boldsymbol{k}(t, x)$, it can be shown using computations similar to Ref. [9] that the operators $\mathcal{I}^{\perp}, \mathcal{H}^{\perp}$ are a compatible bi-Hamiltonian (cosymplectic) pair, while $\mathcal{J}^{\perp}$ is a Hamiltonian symplectic operator. Hence the operator $\mathcal{R}^{\perp}=\mathcal{H}^{\perp} \circ \mathcal{J}^{\perp}$ and its adjoint $\mathcal{R}^{\perp *}=\mathcal{J}^{\perp} \circ \mathcal{H}^{\perp}$ are hereditary recursion operators for the hierarchy of bi-Hamiltonian unconstrained vector potential $\mathrm{mKdV}$ flows including the -1 flow given by the vector SG equation (3.53).

\subsection{Left-invariant frames and square-root operators}

Another choice of non-adapted moving frame $e_{a}$ is available by utilizing the groupmanifold structure that exists for positive constant-curvature spaces in three dimensions. Recall that the compact semisimple Lie group $S U(2)$ is isometric to the 3 -sphere. The isomorphism is expressed via left-invariant vector fields, giving at any point on $N=S^{3}$ an identification of the tangent space $T_{x} S^{3} \simeq \mathfrak{s u}(2) \simeq \mathfrak{s o}(3)$ with the Lie algebra of $S U(2)$, under which the Riemannian metric on $S^{3}$ is identified with the Cartan-Killing 
metric of $\mathfrak{s o}(3)$. Any orthonormal basis in $\mathfrak{s o}(3)$ thereby determines a left-invariant frame $e_{a}$ on the space $N=S^{3}$, satisfying the commutator property

$$
\left[e_{a}, e_{b}\right]=2 \sqrt{\chi} \epsilon_{a b}{ }^{c} e_{c}
$$

and the curvature relation

$$
R\left(e_{c}, e_{d}\right)_{a}{ }^{b}=\chi \epsilon_{c d}{ }^{e} \epsilon_{e a}{ }^{b}=2 \chi \delta_{a[c} \delta_{d]}{ }^{b},
$$

where $\epsilon_{a b c}=\epsilon_{a b}{ }^{d} \delta_{c d}$ (Levi-Civita symbol) represents the $\mathfrak{s o}(3)$ Lie-algebra structure constants, and $\chi>0$ is the curvature scalar of $N=S^{3}$. In contrast to a covariantlyconstant frame, a left-invariant frame is entirely adapted to the group-manifold geometry of the space $N=S^{3}$ and so it is independent of any curves on this space. Nevertheless, such frames will be seen to naturally encode Hamiltonian operators in the frame components of the Cartan structure equations for group-invariant flows of curves $\gamma(t, x)$ on $N=S^{3}$. These flows will be invariant under the group $S O(3)$ of isometries that preserve the Lie algebra structure of $T_{x} S^{3} \simeq \mathfrak{s o}(3)$.

Because of the non-adapted nature of the left-invariant frame, the components $q^{a}$ of the tangent vector $X$ along $\gamma$ are (non-constant) functions of $t, x$. So the most natural Hamiltonian variable will again be $v=X$ while $Y_{\perp}=Y-Y_{\|} X$ and $\varpi=\nabla_{t} X$ continue to be the associated Hamiltonian vector and covector field variables. The flow equation of motion has the same geometrical form as before, given by the part of the torsion structure equation (3.1) projected into the normal space of $\gamma$. However, a crucial difference now is that the curvature structure equation (3.2) becomes redundant since the connection 1 -form determined by the left-invariant frame is just an algebraic expression

$$
w_{a b}=\epsilon_{a b}^{c} e_{c} .
$$

In detail: the covariant derivative of the left-invariant frame in the tangent direction of the curve yields

$$
\nabla_{x} e_{a}=-\frac{1}{2} \sqrt{\chi} e_{a} \times X=-\sqrt{\chi} \epsilon_{a b}^{c} q^{b} e_{c}
$$

and in the flow direction yields

$$
\nabla_{t} e_{a}=-\frac{1}{2} \sqrt{\chi} e_{a} \times Y=-\sqrt{\chi} \epsilon_{a b}{ }^{c} h^{b} e_{c}
$$

giving the connection matrices $\Omega_{a b}=\sqrt{\chi} \epsilon_{a b c} q^{c}$ and $\Theta_{a b}=\sqrt{\chi} \epsilon_{a b c} h^{c}$. Here $\times$ denotes the $\mathfrak{s o}(3)$ Lie-algebra product on $T_{x} S^{3}$. Related to this structure, the frame components of $\varpi^{\prime}=X \times Y$ will be seen to play the role of a Hamiltonian vector field. The flow equation coming from the Cartan structure equations is

$$
q_{t}^{a}=D_{x} h_{\perp}^{a}+h_{\|} q_{x}^{a}+q^{a} h_{\perp b} q_{x}^{b}+2 \sqrt{\chi} \varpi^{\prime a}
$$

in terms of

$$
\varpi^{\prime a}=\epsilon_{b c}^{a} q^{b} h_{\perp}^{c}
$$


where $D_{x} h_{\|}=h_{\perp_{a}} q_{x}^{a}$ is again obtained from the tangential part of the torsion equation, and now

$$
\varpi^{a}=D_{x} h^{a}+\sqrt{\chi} \varpi^{\prime a} .
$$

As before, $q^{a} q_{a}=1$ while $\varpi^{\prime a} q_{a}=\varpi^{a} q_{a}=h_{\perp}{ }^{a} q_{a}=0$, and the same 3 -component vector notation will be employed hereafter. Consequently, the flow equation takes the Hamiltonian form

$$
\vec{q}_{t}=\mathcal{H}\left(\vec{h}_{\perp}\right)+2 \sqrt{\chi} \mathcal{I}\left(\vec{h}_{\perp}\right)
$$

where the operator

$$
\mathcal{H}=D_{x}+\vec{q}_{x} D_{x}^{-1}\left(\vec{q}_{x} \cdot\right)+\vec{q}\left(\vec{q}_{x} \cdot\right)
$$

is unchanged, but now

$$
\mathcal{I}=\vec{q} \times
$$

is an algebraic operator (with the $\mathfrak{s o}(3)$ Lie-algebra product identified with the vector cross-product) satisfying $\mathcal{I}^{2}=\vec{q} \times(\vec{q} \times)=-1+\vec{q}(\vec{q} \cdot)=-\mathrm{id}_{\perp}$. Omitting details, it can be proved that these operators enjoy the same properties as the Hamiltonian operators (3.32) and (3.31) in the covariantly-constant frame.

Theorem 5: $\mathcal{I}, \mathcal{H}$ are a pair of compatible Hamiltonian (cosymplectic) operators and obey $\vec{q} \cdot \mathcal{I}=\vec{q} \cdot \mathcal{H}=0$, with respect to the constrained Hamiltonian variable $\vec{q}(t, x)$. The inverse of $\mathcal{I}$ is a Hamiltonian symplectic operator $\mathcal{J}=\mathcal{I}^{-1}=-\vec{q} \times=-\mathcal{I}$ whose domain is defined on the $x$-jet space coordinates $\perp \vec{q}$. $\ddagger$ Then the flow equation on $\vec{q}$ becomes $\vec{q}_{t}=2 \sqrt{\chi} \vec{\varpi}^{\prime}+\mathcal{R}\left(\vec{\varpi}^{\prime}\right)$ in terms of the operator $\mathcal{R}=\mathcal{H} \circ \mathcal{J}$, where $\vec{\varpi}^{\prime}=\mathcal{I}\left(\vec{h}_{\perp}\right)$ is the inverse image of $\vec{\varpi}=\mathcal{H}\left(\vec{h}_{\perp}\right)+\sqrt{\chi} \mathcal{I}\left(\vec{h}_{\perp}\right)$ under the shifted operator $\mathcal{R}^{\prime}=\mathcal{R}+\sqrt{\chi}$, namely $\vec{\varpi}=\mathcal{R}^{\prime}\left(\vec{\varpi}^{\prime}\right)$.

On the $x$-jet space of $\vec{q}(t, x)$, it follows that $\vec{\varpi} \cdot \partial / \partial \vec{q}$ has the geometrical meaning of a Hamiltonian vector field as does $\vec{\varpi}^{\prime} \cdot \partial / \partial \vec{q}$, and $\vec{h}_{\perp} \cdot d \vec{q}$ has the geometrical meaning of a variational covector field. Hence $\mathcal{R}=\mathcal{H} \circ \mathcal{J}$ and its adjoint $\mathcal{R}^{*}=\mathcal{J} \circ \mathcal{H}$ will be hereditary recursion operators for respective hierarchies of commuting Hamiltonian vector fields $\vec{\omega}^{(k)}$ and involutive variational covector fields $\vec{h}_{\perp}{ }^{(k)}, k=0,1,2, \ldots$, related by $\vec{h}_{\perp}{ }^{(k)}=\mathcal{J}\left(\vec{\varpi}^{(k)}\right)$. Because $\mathcal{J}=-\mathcal{I}$ is purely an algebraic operator, these recursion operators are like a "square root" of the ones obtained from the covariantly-constant frame. The hierarchy starts at

$$
\vec{\varpi}^{(0)}=\vec{q}_{x}
$$

describing infinitesimal $x$-translations in terms of the arclength $x$ along the curve $\gamma$, while

$$
\vec{h}_{\perp}{ }^{(0)}=\mathcal{J}\left(\vec{q}_{x}\right)=-\vec{q} \times \vec{q}_{x} .
$$

$\ddagger$ Namely, the coordinate space $\left\{\left(\vec{q}_{x}, \vec{q}_{x x}, \ldots\right)_{\perp}=\left(\vec{q}_{x}, \vec{q}_{x x}+\left|\vec{q}_{x}\right|^{2} \vec{q}, \ldots\right)\right\}$ consisting of those vectors $\perp$ $\vec{q}$ derived from the differential consequences of $|\vec{q}|^{2}=1$. 
Next comes

$$
\vec{\varpi}^{(1)}=\mathcal{R}\left(\vec{q}_{x}\right)=-\mathcal{H}\left(\vec{q} \times \vec{q}_{x}\right)=-\vec{q} \times \vec{q}_{x x}
$$

and

$$
\vec{h}_{\perp}^{(1)}=\mathcal{J}\left(-\vec{q} \times \vec{q}_{x}\right)=-\left(\vec{q}_{x x}+\left|\vec{q}_{x}\right|^{2} \vec{q}\right),
$$

followed by

$$
\vec{\varpi}^{(2)}=-\vec{q}_{x x x}-\frac{3}{2}\left(\left|\vec{q}_{x}\right|^{2} \vec{q}\right)_{x}
$$

and

$$
\vec{h}_{\perp}^{(2)}=-\vec{q} \times \vec{q}_{x x x}-\frac{3}{2}\left|\vec{q}_{x}\right|^{2} \vec{q} \times \vec{q}_{x} .
$$

In particular, the vector fields $\vec{\varpi}^{(0)}, \vec{\varpi}^{(2)}$, and so on for even $k$, along with the covector fields $\vec{h}_{\perp}{ }^{(1)}, \vec{h}_{\perp}{ }^{(3)}$, and so on for odd $k$, exactly reproduce the hierarchy of potential mKdV Hamiltonian vector and covector fields with respect to $\vec{q}(t, x)$ derived in the covariantly-constant frame.

The full hierarchy in the left-invariant frame has a natural bi-Hamiltonian structure $\vec{\varpi}^{(k)}=\mathcal{I}\left(\vec{h}_{\perp}{ }^{(k)}\right)=\mathcal{H}\left(\vec{h}_{\perp}{ }^{(k-1)}\right)$ with Hamiltonians $H=H^{(k)}\left(\vec{q}, \vec{q}_{x}, \vec{q}_{x x}, \ldots\right)$ determined by $\vec{h}_{\perp}{ }^{(k)}=\delta H^{(k)} / \delta \vec{q}$ subject to Lagrangian multipliers enforcing the constraint condition $\vec{q} \cdot \delta H / \delta \vec{q}=0$ like before. Thus, modulo the Lagrangian multipliers,

$$
H^{(1)}=\frac{1}{2}\left|\vec{q}_{x}\right|^{2}
$$

agrees with the bottom Hamiltonian in the hierarchy in the covariantly-constant frame, while the next Hamiltonian in the hierarchy here is given by

$$
H^{(2)}=-\frac{1}{2} \vec{q} \cdot\left(\vec{q}_{x} \times \vec{q}_{x x}\right),
$$

and so on to all higher orders $k=1,2, \ldots$ But there is no Hamiltonian $H^{(0)}$ since any scalar triple product expressions that can be formed just out of $\vec{q}$ and $\vec{q}_{x}$ are trivial.

The entire hierarchy $k=0,1,2, \ldots$, produces commuting flows

$$
\vec{q}_{t}=2 \sqrt{\chi} \vec{\varpi}^{(k)}+\vec{\varpi}^{(k+1)},
$$

with $|\vec{q}|=1$. These flows describe constrained vector PDEs having a bi-Hamiltonian structure

$$
\vec{q}_{t}=\mathcal{I}\left(\delta H^{(k, \chi)} / \delta \vec{q}\right)=\mathcal{H}\left(\delta H^{(k-1, \chi)} / \delta \vec{q}\right)
$$

given by the Hamiltonians

$$
H^{(k, \chi)}=2 \sqrt{\chi} H^{(k)}+H^{(k+1)} .
$$

The form of the PDEs here is $S O(3)$-invariant, expressed by covariant vector functions $\vec{\varpi}^{\prime}=\vec{\varpi}^{(k)}\left(\vec{q}, \vec{q}_{x}, \vec{q}_{x x}, \ldots\right)$ and $\vec{h}_{\perp}=\vec{h}_{\perp}{ }^{(k)}\left(\vec{q}, \vec{q}_{x}, \vec{q}_{x x}, \ldots\right)$ consisting of explicit polynomials constructed using vector dot products and cross products on the $x$-jet space of $\vec{q}(t, x)$. Compared to the larger $O(3)$-invariance in the covariantly-constant frame, invariance 
under reflections on $\vec{q}$ in the left-invariant frame is lost due to the explicit appearance of the cross-product operator.

There is a corresponding hierarchy of group-invariant non-stretching motions

$$
\gamma_{t}=f\left(\gamma_{x}, \nabla_{x} \gamma_{x}, \nabla_{x}^{2} \gamma_{x}, \ldots\right)
$$

on the underlying curve $\gamma(t, x)$. These motions are obtained through substitution of

$$
\begin{aligned}
& q^{a} e_{a}=\gamma_{x}, \\
& q^{a} \nabla_{x} e_{a}=0, \\
& q_{x}^{a} e_{a}=\nabla_{x}\left(q^{a} e_{a}\right)-q^{a} \nabla_{x} e_{a}=\nabla_{x} \gamma_{x}, \\
& q_{x}^{a} \nabla_{x} e_{a}=\frac{1}{2} \sqrt{\chi} \gamma_{x} \times \nabla_{x} \gamma_{x}=-\frac{1}{2} \epsilon_{a b}^{c} q_{x}^{a} q^{b} e_{c},
\end{aligned}
$$

and so on, into the flow equation (3.80) given by $f=h^{a} e_{a}$ with $h^{a}=h_{\|} q^{a}+h_{\perp}{ }^{a}$ and $h_{\|}=D_{x}^{-1}\left(h_{\perp_{a}} q_{x}^{a}\right)$. The 0 flow on $\vec{q}$ producing the vector PDE

$$
\vec{q}_{t}=-\vec{q} \times \vec{q}_{x x}+2 \sqrt{\chi} \vec{q}_{x}
$$

yields the curve motion

$$
\gamma_{t}=\gamma_{x} \times \nabla_{x} \gamma_{x}
$$

This motion defines a geometric map equation on the group-manifold $N=S^{3}$ and will be called the $S O(3)$ group-motion equation for $\gamma$. A linear combination of this equation (3.86) and the non-stretching mKdV map equation (3.25) gives the curve motion determined by the +1 flow

$$
\vec{q}_{t}=-\vec{q}_{x x x}-\frac{3}{2}\left(\left|\vec{q}_{x}\right|^{2} \vec{q}\right)_{x}-2 \sqrt{\chi} \vec{q} \times \vec{q}_{x x} .
$$

Conversely the non-stretching mKdV map equation is given by a linear combination of the 0 flow and +1 flow equations. A similar result holds for the hierarchy of higher order non-stretching mKdV map equations when expressed as vector PDEs for $\vec{q}$ in the left-invariant frame as follows.

Through the geometrical meaning of $\vec{h}_{\perp}$ and $\vec{\omega}$ as the respective frame components of the normal motion $\left(\gamma_{t}\right)_{\perp}$ of the curve and the evolution of its tangent vector $\nabla_{t} \gamma_{x}$, any constrained potential $\mathrm{mKdV}$ flow in the covariantly-constant frame describes a non-stretching curve motion corresponding to a flow in the left-invariant frame (and conversely) via replacing $D_{x} \leftrightarrow \mathcal{D}_{x}$ in the expressions for the Hamiltonian vector fields $\vec{\varpi}\left(\vec{q}, \vec{q}_{x}, \vec{q}_{x x}, \ldots\right)$ in the two frames, where

$$
\mathcal{D}_{x}=D_{x}+\sqrt{\chi} \vec{q} \times
$$

This correspondence arises geometrically from the relation between the frame equations $0=\nabla_{x} e_{a}$ in the covariantly-constant case and $0=\left(\nabla_{x}+\sqrt{\chi} \gamma_{x} \times\right) e_{a}$ in the left-invariant case. The shifted recursion operator then has the interpretation as a covariantized operator given by $\mathcal{R} \leftrightarrow \mathcal{R}^{\prime}$ under replacing $D_{x} \leftrightarrow \mathcal{D}_{x}$ in the Hamiltonian operator $\mathcal{H}$. 
There is an analogous correspondence relating the flows in the left-invariant frame to the flows in the parallel frame via the relations

$$
\begin{aligned}
& \vec{q} \leftrightarrow \gamma_{x} \leftrightarrow(\mathbf{0}, 1), \\
& \mathcal{D}_{x} \vec{q}=\vec{q}_{x} \leftrightarrow \nabla_{x} \gamma_{x} \leftrightarrow(\boldsymbol{v}, 0), \\
& \mathcal{D}_{x} \vec{q}_{x} \leftrightarrow \nabla_{x}^{2} \gamma_{x} \leftrightarrow \boldsymbol{D}_{x}(\boldsymbol{v}, 0),
\end{aligned}
$$

and so on, with

$$
\boldsymbol{D}_{x}=D_{x}+(* \boldsymbol{v}, 0) \times
$$

while

$$
\vec{q} \times \leftrightarrow * \leftrightarrow(\mathbf{0}, 1) \times,
$$

similarly to the correspondence for the case of the covariantly-constant frame.

Theorem 6: For any flow $\vec{q}_{t}=\vec{\varpi}^{(\chi)}\left(\vec{q}, \vec{q}_{x}, \vec{q}_{x x}, \ldots\right)$ in the left-invariant frame (where $\vec{\varpi}^{(\chi)}$ denotes a linear combination of Hamiltonian vector fields $\vec{\varpi}^{(k+1)}+2 \sqrt{\chi} \vec{\varpi}^{(k)}$, $k=0,1, \ldots)$, there is a geometrically corresponding flow in the parallel frame, $\boldsymbol{v}_{t}=\tilde{\mathcal{H}}^{(\chi)}\left(\varpi^{(\chi)}\left((\mathbf{0}, 1),(\boldsymbol{v}, 0), \boldsymbol{D}_{x}^{(\chi)}(\boldsymbol{v}, 0), \ldots\right)\right)=\boldsymbol{h}_{\perp}{ }^{(\chi)}\left(\boldsymbol{v}, \boldsymbol{v}_{x}, \ldots\right)$ in terms of the $m K d V$ Hamiltonian operator $\tilde{\mathcal{H}}=D_{x}+* \boldsymbol{v} D_{x}^{-1}(* \boldsymbol{v} \cdot)$, where $\boldsymbol{D}_{x}^{(\chi)}=\boldsymbol{D}_{x}-\sqrt{\chi} *$ and $\tilde{\mathcal{H}}^{(\chi)}=\tilde{\mathcal{H}}-\sqrt{\chi} *$. Moreover, the shifted recursion operator in the left-invariant frame corresponds exactly to the square root of the $m K d V$ recursion operator,

$$
\mathcal{R}^{\prime 2}=-\tilde{\mathcal{H}} \circ \tilde{\mathcal{J}}
$$

and

$$
\mathcal{R}^{\prime}=* \tilde{\mathcal{J}}=\tilde{\mathcal{H}} *
$$

where $\tilde{\mathcal{J}}=D_{x}+\boldsymbol{v} D_{x}^{-1}(\boldsymbol{v} \cdot)$ is the $m K d V$ symplectic operator.

The Hodge-star operator $*$ here plays the role in the parallel frame of an additional Hamiltonian operator $\tilde{\mathcal{I}}=-*$ which is compatible with $\tilde{\mathcal{H}}$ and corresponds to the symplectic operator $\mathcal{J}$ in the left-invariant frame. Under this correspondence, the $S O(3)$ group-motion equation (3.86) is given by the flow

$$
\boldsymbol{v}_{t}=*\left(\boldsymbol{v}_{x x}+\frac{1}{2}|\boldsymbol{v}|^{2} \boldsymbol{v}+\chi \boldsymbol{v}\right)
$$

with

$$
\boldsymbol{h}_{\perp}=* \boldsymbol{v},
$$

which is outside the standard mKdV hierarchy. In particular, the square-root recursion operator $\mathcal{R}^{\prime}$ generates an enlarged hierarchy of flows

$$
\boldsymbol{v}_{t}=\mathcal{R}^{\prime k}(* \boldsymbol{v})=\boldsymbol{h}_{\perp}{ }^{(k)}, \quad k=0,1, \ldots,
$$

commuting with the mKdV flows. These flows (3.98) inherit a bi-Hamiltonian structure from their counterparts in the left-invariant frame,

$$
\boldsymbol{v}_{t}=\tilde{\mathcal{H}}\left(\varpi^{(k-1)}\right)=\tilde{\mathcal{I}}\left(\varpi^{(k)}\right)
$$


in terms of the adjoint recursion operator $\mathcal{R}^{\prime *}=\tilde{\mathcal{J}} *=* \tilde{\mathcal{H}}$, where

$$
\varpi^{(k)}=\mathcal{R}^{\prime * k}(\boldsymbol{v})=* \boldsymbol{h}_{\perp}{ }^{(k)} .
$$

Associated to this structure are commuting Hamiltonian vector fields $\boldsymbol{h}_{\perp}{ }^{(k)} \cdot \partial / \partial \boldsymbol{v}$ and involutive covector fields $\varpi^{(k)} \cdot d \boldsymbol{v}, k=0,1, \ldots$, from which the Hamiltonians $H^{(k)}$ are determined by

$$
\delta H^{(k)} / \delta \boldsymbol{v}=* \boldsymbol{h}_{\perp}{ }^{(k)}=\varpi^{(k)} .
$$

In detail, the hierarchy looks like:

$$
\begin{aligned}
& \varpi^{(0)}=\boldsymbol{v}, \quad H^{(0)}=\frac{1}{2}|\boldsymbol{v}|^{2} \\
& \varpi^{(1)}=* \boldsymbol{v}_{x}, \quad H^{(1)}=\frac{1}{2} \boldsymbol{v} \cdot * \boldsymbol{v}_{x} ; \\
& \varpi^{(2)}=-\boldsymbol{v}_{x x}-\frac{1}{2}|\boldsymbol{v}|^{2} \boldsymbol{v}, \quad H^{(2)}=\frac{1}{2}\left|\boldsymbol{v}_{x}\right|^{2}+\frac{1}{8}|\boldsymbol{v}|^{4} ; \\
& \varpi^{(3)}=-*\left(\boldsymbol{v}_{x x x}+\frac{3}{2}|\boldsymbol{v}|^{2} \boldsymbol{v}\right), \quad H^{(3)}=\frac{1}{2} \boldsymbol{v}_{x} \cdot * \boldsymbol{v}_{x x}-\frac{3}{8}|\boldsymbol{v}|^{2} \boldsymbol{v} \cdot * \boldsymbol{v}_{x},
\end{aligned}
$$

and so on.

This enlarged hierarchy contains the same -1 flow as in the mKdV hierarchy, which arises directly from the kernel of the square-root recursion operator $\mathcal{R}^{\prime}$. In the left-invariant frame, this flow is given by $0=\vec{\varpi}=\mathcal{H}\left(\vec{h}_{\perp}\right)+\sqrt{\chi} \mathcal{I}\left(\vec{h}_{\perp}\right)=\mathcal{D}_{x} \vec{h}$ where $\vec{h}=\vec{h}_{\perp}+h_{\|} \vec{q}$ is a covariantly-constant vector, with $D_{x} h_{\|}=\vec{h}_{\perp} \cdot \vec{q}_{x}$. Note $h_{\|}$and $\vec{h}_{\perp}$ are related through the conservation law $0=D_{x}|\vec{h}|^{2}$, with the consequence that $|\vec{h}|$ depends only on $t$ and hence satisfies $1=|\vec{h}|^{2}=\left|\vec{h}_{\perp}\right|^{2}+h_{\|}^{2}$ after $t$ is conformally scaled. The -1 flow equation produced on $\vec{q}$ is then given by

$$
\vec{q}_{t}=\sqrt{\chi} \vec{\varpi}^{\prime}=\sqrt{\chi} \vec{q} \times \vec{h}_{\perp}
$$

or simply

$$
\mathcal{D}_{t} \vec{q}=0
$$

so thus $\vec{q}$ is covariantly constant with respect to the covariant derivative

$$
\mathcal{D}_{t}=D_{t}+\sqrt{\chi} \vec{h} \times
$$

These equations in covariant-derivative form on $\vec{q}$ and $\vec{h}$ together are recognized to be a constrained vector $\mathfrak{s u}(2)$ chiral model [27, which is geometrically equivalent to the nonstretching wave map equation $\varpi=\nabla_{t} \gamma_{x}=0$ on the group-manifold $N=S U(2) \simeq S^{3}$, for the curve $\gamma(t, x)$ subject to $1=\left|\gamma_{x}\right|_{g}=\left|\gamma_{t}\right|_{g}$.

Interestingly, the wave map equation describing the -1 flow can be expressed as a vector hyperbolic equation on $\vec{q}(t, x)$, via eliminating $\vec{h}_{\perp}=-\sqrt{\chi} \vec{q} \times \vec{q}_{t}$ and

$$
\begin{aligned}
h_{\|}=\sqrt{1-\left|\vec{h}_{\perp}\right|^{2}} & =\sqrt{1-\chi^{-1}\left|\vec{q}_{t}\right|^{2}} \text { to get } \\
\vec{q}_{t x} & =-\vec{q}_{t} \cdot \overrightarrow{q_{x}} \vec{q}-\vec{q} \times\left(\vec{q}_{t}+\sqrt{1-\left|\vec{q}_{t}\right|^{2}} \vec{q}_{x}\right)
\end{aligned}
$$

after $\chi$ is scaled out by $t \rightarrow t / \sqrt{\chi}, x \rightarrow x / \sqrt{\chi}$. 
Another formulation of the -1 flow equation is provided by a covariant-derivative vector SG equation derived as follows for the 2-component vector variable $\boldsymbol{h}_{\perp}(t, x)$. First, differentiation of $0=\mathcal{D}_{t} \vec{q}$ by $\mathcal{D}_{x}$ followed by use of the commutator

$$
\left[\mathcal{D}_{x}, \mathcal{D}_{t}\right]=\chi(\vec{q} \vec{h} \cdot-\vec{h} \vec{q} \cdot)
$$

gives $0=\mathcal{D}_{t} \vec{q}_{x}-\chi \vec{h}_{\perp}$. Next, $\vec{q}_{x}$ can be eliminated in terms of $h_{\|}$and $\vec{h}_{\perp}$ from $0=\mathcal{D}_{x} \vec{h}=\mathcal{D}_{x}^{\perp} \vec{h}_{\perp}+h_{\|} \vec{q}_{x}$ obtained by decomposition of $\vec{h}$ and $\mathcal{D}_{x}$ into tangential and normal parts relative to $\vec{q}$, where the derivative operator

$$
\mathcal{D}_{x}^{\perp}=D_{x}^{\perp}+\sqrt{\chi} \vec{q} \times
$$

satisfies $\vec{q} \cdot \mathcal{D}_{x}^{\perp}=\vec{q} \cdot D_{x}^{\perp}=0$. This operator has a natural meaning through the geometrical correspondence

$$
\boldsymbol{v} \leftrightarrow \nabla_{x} \gamma_{x} \leftrightarrow \vec{q}_{x}, \quad \boldsymbol{v}_{x} \leftrightarrow\left(\nabla_{x}^{2} \gamma_{x}\right)_{\perp} \leftrightarrow \mathcal{D}_{x}^{\perp} \vec{q}_{x},
$$

and so on, between the parallel frame and left-invariant frame. Finally, through the derivative operator

$$
\mathcal{D}_{t}^{\perp}=D_{t}^{\perp}+\sqrt{\chi}\left(\vec{h}_{\perp} \times+h_{\|} \vec{q} \times\right)
$$

which obeys $\vec{q} \cdot \mathcal{D}_{t}^{\perp}=0$, we have the equation

$$
-\mathcal{D}_{t}^{\perp}\left(h_{\|}^{-1} \mathcal{D}_{x}^{\perp} \boldsymbol{h}_{\perp}\right)=\chi \boldsymbol{h}_{\perp},
$$

where $h_{\|}=\sqrt{1-\left|\boldsymbol{h}_{\perp}\right|^{2}}$.

Theorem 7: In positive constant-curvature spaces $(N, g) \simeq S^{3} \simeq S U(2)$ in three dimensions, the group-invariant integrable flows of curves $\gamma(t, x)$ comprise an enlarged hierarchy whose even-order flows consist of the $S O(3)$ group-motion equation

$$
\gamma_{t}=\gamma_{x} \times \nabla_{x} \gamma_{x}
$$

and higher order analogs, and whose odd-order flows consist of the non-stretching $m K d V$ map equation

$$
\gamma_{t}=\nabla_{x}^{2} \gamma_{x}+\frac{3}{2}\left|\nabla_{x} \gamma_{x}\right|_{g}^{2} \gamma_{x}
$$

and higher order analogs, all subject to $\left|\gamma_{x}\right|_{g}=1$. The -1 flow is the non-stretching wave map equation

$$
\nabla_{t} \gamma_{x}=0, \quad\left|\gamma_{x}\right|_{g}=\left|\gamma_{t}\right|_{g}=1
$$

which is equivalent to the $S U(2)$ chiral model.

Corollary: The $-1,0,+1$ flows can be reformulated as motions of an arclengthparameterized embedded curve in $\mathbb{R}^{4}$ described by a 4-component vector variable $\vec{u}(t, x)$. This vector is constrained such that $|\vec{u}|=1$ so the curve motion is confined to the 3 sphere $S^{3} \simeq S U(2)$, and in addition $\left|\vec{u}_{x}\right|=1$ so that the curve does not stretch (namely $x$ is arclength). Under the resulting identifications

$$
\gamma \leftrightarrow \vec{u}, \quad \nabla_{x} \leftrightarrow D_{x}-\vec{u}\left(\vec{u} \cdot D_{x}\right),
$$

the wave map equation becomes the $O(4)$ sigma model

$$
\vec{u}_{t x}=-\vec{u}_{t} \cdot \vec{u}_{x} \vec{u}
$$


the mKdV map equation reduces to the model

$$
\vec{u}_{t}=\vec{u}_{x x x}+\frac{3}{2} \vec{u}_{x x} \cdot \vec{u}_{x x} \vec{u}_{x}
$$

up to a convective term; and the $S O(3)$ group-motion equation is given by the model

$$
\vec{u}_{t}=*\left(\vec{u} \wedge \vec{u}_{x} \wedge \vec{u}_{x x}\right) .
$$

Here $\cdot$ is the dot product, $\wedge$ the wedge product, and $*$ the Hodge-star operator, on $\mathbb{R}^{4}$.

\section{Hamiltonian operators and integrable flows of curves in higher dimensions}

The derivation of bi-Hamiltonian operators from frame formulations of flows of nonstretching curves in three-dimensional spaces $S^{3} \simeq S U(2), H^{3}, \mathbb{R}^{3}$ generalizes to all higher dimensions both for the case of constant-curvature spaces and Lie-group spaces. As in three-dimensions, the flow of a curve $\gamma(t, x)$ in an $n$-dimensional Riemannian manifold $(N, g)$ for any $n \geq 2$ has associated to it the Cartan structure equations expressing curvature $\left[\nabla_{x}, \nabla_{t}\right]=R\left(\gamma_{x}, \gamma_{t}\right)$ and torsion $\nabla_{x} \gamma_{t}-\nabla_{t} \gamma_{x}=\left[\gamma_{x}, \gamma_{t}\right]=0$ in terms of orthonormal frame vectors $e_{a}$ and connection 1-forms $w_{a b}$ in the tangent space $T_{\gamma} N$. The same natural encoding of a compatible pair of Hamiltonian operators contained in these equations is seen in all dimensions, through the use of a geometrical choice of $e_{a}$ and $w_{a b}$ given by a covariantly-constant frame or a parallel frame if $N$ is a constant-curvature space, or a left-invariant frame if $N$ is a Lie-group space. For such frames the curvature matrix will be constant on $N$, leading to a simple bi-Hamiltonian structure based on the geometric variables given by the tangent vector $X=\gamma_{x}=q^{a} e_{a}$ along $\gamma$, the flow vector $Y_{\perp}=\left(\gamma_{t}\right)_{\perp}=h_{\perp}{ }^{a} e_{a}$ normal to $\gamma$, and the principal normals $v=\nabla_{x} X=v^{a} e_{a}$ and $\varpi=\nabla_{t} X=\varpi^{a} e_{a}$ associated with the tangential and flow directions of $\gamma$, where $|X|_{g}=1$ and $g(v, X)=g(\varpi, X)=0$ for a non-stretching curve $\gamma$. Note the frame components of the principal normals $v$, $\varpi$ will be differential covariants of $\gamma$.

\subsection{Constant-curvature spaces}

Let $(N, g)$ be an $n$-dimensional constant-curvature Riemannian manifold $(n \geq 2)$, with curvature scalar $\chi$. The curvature matrix for any orthonormal frame $e_{a}$ is simply

$$
R\left(e_{c}, e_{d}\right)_{a}^{b}=2 \chi \delta_{a[c} \delta_{d]}^{b} .
$$

Recall a parallel frame is adapted to $\gamma$ via

$$
e_{a}=\left(\left(e_{a}\right)_{\perp}, X\right)
$$

such that the covariant derivative of the normal vectors in the frame

$$
\nabla_{x}\left(e_{a}\right)_{\perp}=-v_{a} X
$$

is tangential to $\gamma$ and the covariant derivative of the tangent vector

$$
\nabla_{x} X=v^{a}\left(e_{a}\right)_{\perp}
$$


is normal to $\gamma$ (where, note, $q^{a}=$ const). The resulting Cartan structure equations carry over directly from three dimensions to $n$ dimensions where $v^{a}, \varpi^{a}, h_{\perp}{ }^{a}$ now are $n-1$ component vectors. Thus, the flow equation on $v^{a}$ retains the form (using index-free notation)

$$
\boldsymbol{v}_{t}=\mathcal{H}(\varpi)+\chi \mathcal{I}(\varpi)=\boldsymbol{h}_{\perp}^{\prime}+\chi \boldsymbol{h}_{\perp}
$$

where

$$
\left.\mathcal{H}=D_{x}+\boldsymbol{v}\right\lrcorner D_{x}^{-1}(\boldsymbol{v} \wedge), \quad \mathcal{I}^{-1}=\mathcal{J}=D_{x}+\boldsymbol{v} D_{x}^{-1}(\boldsymbol{v} \cdot)
$$

are, respectively, Hamiltonian cosymplectic and symplectic operators and $\mathcal{R}=\mathcal{H} \circ \mathcal{J}$ is a hereditary recursion operator as proved in Ref. [9]. Here $\boldsymbol{h}_{\perp}=\mathcal{I}(\varpi)$ and its image $\boldsymbol{h}_{\perp}^{\prime}=\mathcal{H}(\varpi)$ under $\mathcal{R}$ have the roles of Hamiltonian vector fields with respect to $\boldsymbol{v}(t, x)$, while the role of $\varpi$ is a covector field. (Compared with Ref. [9] the present formulation of these operators is manifestly $O(n-1)$-invariant, employing just the vector dot product, interior and exterior vector products. In particular, $\boldsymbol{C}\lrcorner(\boldsymbol{B} \wedge \boldsymbol{A})=(\boldsymbol{C} \cdot \boldsymbol{B}) \boldsymbol{A}-(\boldsymbol{C} \cdot \boldsymbol{A}) \boldsymbol{B}$.)

The resulting hierarchy of flows generated by $\mathcal{R}$ has the same structure as in three dimensions: At the bottom the 0 flow is given by $\boldsymbol{h}_{\perp}=\boldsymbol{v}_{x}$, with $\boldsymbol{v}$ seen to satisfy the vector mKdV equation

$$
\boldsymbol{v}_{t}=\boldsymbol{v}_{x x x}+\frac{3}{2}|\boldsymbol{v}|^{2} \boldsymbol{v}_{x}+\chi \boldsymbol{v}_{x}
$$

up to a convective term. The non-stretching curve motion of $\gamma$ produced by this flow is a corresponding mKdV map equation

$$
\gamma_{t}=\nabla_{x}^{2} \gamma_{x}+\frac{3}{2}\left|\nabla_{x} \gamma_{x}\right|_{g}^{2} \gamma_{x}
$$

with $\left|\gamma_{x}\right|_{g}=1$. There is a -1 flow mapped into $\boldsymbol{h}_{\perp}^{\prime}=0$ under $\mathcal{R}$ in the hierarchy, which is given by the kernel of $\mathcal{H}$ determined by $\varpi=0$. This yields a nonlocal flow $\boldsymbol{h}_{\perp}=-D_{x}^{-1}\left(h_{\|} \boldsymbol{v}\right)$, with the conservation law $D_{x}\left(\left|\boldsymbol{h}_{\perp}\right|^{2}+h_{\|}^{2}\right)=0$ implying that the flow is conformally equivalent to one with uniform speed. Thus, after a conformal scaling of $t$ is made, $\boldsymbol{v}$ in this flow then satisfies the vector hyperbolic equation

$$
\boldsymbol{v}_{t x}=-\sqrt{1-\left|\boldsymbol{v}_{t}\right|^{2}} \boldsymbol{v}
$$

Equivalently, $\boldsymbol{h}_{\perp}$ obeys the vector $\mathrm{SG}$ equation

$$
\left(\sqrt{\left(1-\left|\boldsymbol{h}_{\perp}\right|^{2}\right)^{-1}} \boldsymbol{h}_{\perp x}\right)_{t}=-\boldsymbol{h}_{\perp} .
$$

The corresponding curve motion of $\gamma$ is the non-stretching wave map equation

$$
\nabla_{t} \gamma_{x}=0, \quad\left|\gamma_{x}\right|_{g}=\left|\gamma_{t}\right|_{g}=1
$$

as seen from the geometrical meaning of $\varpi$.

A covariantly-constant frame provides a different formulation of these main results, arising in terms of vector potential variables. Recall, since $\nabla_{x} e_{a}=0$, the principal normal for the tangential direction of $\gamma$ for a covariantly-constant frame is given by $v^{a}=q_{x}^{a}$ where $q^{a} \neq$ const. In the Cartan structure equations in $n$ dimensions, $q^{a},{h_{\perp}}^{a}, \varpi^{a}$ now are $n$-component vectors, with $q^{a}$ having unit norm and with $h_{\perp}{ }^{a}, \varpi^{a}$ 
$\perp$ to $q^{a}$. Compared to three dimensions, vector cross products are replaced by interior and exterior products, so thus the flow equation on $q^{a}$ becomes

$$
\vec{q}_{t}=\chi \mathcal{I}\left(\vec{h}_{\perp}\right)+\mathcal{H}\left(\vec{h}_{\perp}\right)=\chi \vec{\varpi}^{\prime}+\vec{\varpi}
$$

where

$$
\mathcal{H}=D_{x}+\vec{q}_{x} D_{x}^{-1}\left(\vec{q}_{x} \cdot\right)+\vec{q}\left(\vec{q}_{x} \cdot\right)
$$

as before, while now

$$
\mathcal{I}=-\vec{q}\lrcorner D_{x}^{-1}(\vec{q} \wedge) .
$$

The main results on these operators carry over to $n$ dimensions: $\mathcal{I}, \mathcal{H}$ are a pair of compatible Hamiltonian (cosymplectic) operators obeying $\vec{q} \cdot \mathcal{I}=\vec{q} \cdot \mathcal{H}=0$ with respect to the constrained variable $\vec{q}(t, x)$, and so $\mathcal{J}=\mathcal{I}^{-1}$ defines a symplectic operator. (Note the domain of these operators consists of the $x$-jet space coordinates $\perp \vec{q}$. $\ddagger)$ The role of Hamiltonian vector fields is hence played by $\vec{\varpi}=\mathcal{H}\left(\vec{h}_{\perp}\right)$ and its inverse image $\vec{\varpi}^{\prime}=\mathcal{I}\left(\vec{h}_{\perp}\right)$ under the hereditary recursion operator $\mathcal{R}=\mathcal{H} \circ \mathcal{J}$. In dimensions $n>3$, however, there is a noteworthy difference that an explicit simple expression for the inverse of $\mathcal{I}$ can no longer be derived.

The hierarchy of flows in the parallel frame corresponds to a hierarchy of constrained-potential flows in the covariantly-constant frame as a result of the geometrical meaning of the frame components $\vec{h}_{\perp}$ for the normal motion $\left(\gamma_{t}\right)_{\perp}$ of the curve as well as $\vec{\varpi}$ for the evolution of the tangent vector $\nabla_{t} X$ of the curve. It is natural to express this correspondence by first resolving the constraint $|\vec{q}|=1$ through splitting

$$
\vec{q}=(k, \boldsymbol{k})
$$

into a scalar variable $k$ and a $n-1$-component vector variable $\boldsymbol{k}$ defined relative to any fixed unit vector $\hat{n}$, so

$$
k=\sqrt{1-|\boldsymbol{k}|^{2}} .
$$

This splitting yields the relations

$$
\begin{aligned}
& \vec{q}_{x}=\left(-k^{-1} \boldsymbol{k} \cdot \boldsymbol{k}_{x}, \boldsymbol{k}_{x}\right), \\
& D_{x}^{\perp} \vec{q}_{x}=\left(-k^{-1} \boldsymbol{k} \cdot D_{x}^{\perp} \boldsymbol{k}_{x}, D_{x}^{\perp} \boldsymbol{k}_{x}\right),
\end{aligned}
$$

and so on, where

$$
D_{x}^{\perp}=D_{x}+\boldsymbol{k} k\left(\left(k^{-1} \boldsymbol{k}\right)_{x} \cdot\right)
$$

which comes from a similar splitting of $D_{x}^{\perp}=D_{x}+\vec{q}\left(\vec{q}_{x} \cdot\right)$. Hence the correspondence looks like

$$
\boldsymbol{v} \leftrightarrow \nabla_{x} \gamma_{x} \leftrightarrow \boldsymbol{k}_{x}, \quad \boldsymbol{v}_{x} \leftrightarrow\left(\nabla_{x}^{2} \gamma_{x}\right)_{\perp} \leftrightarrow D_{x}^{\perp} \boldsymbol{k}_{x}
$$

$\ddagger$ Namely, the coordinate space $\left\{\left(\vec{q}_{x}, \vec{q}_{x x}, \ldots\right)_{\perp}=\left(\vec{q}_{x}, \vec{q}_{x x}+\left|\vec{q}_{x}\right|^{2} \vec{q}, \ldots\right)\right\}$ consisting of those vectors $\perp$ $\vec{q}$ derived from the differential consequences of $|\vec{q}|^{2}=1$. 
and so on. In particular, as in three dimensions, the 0 flow on $\boldsymbol{k}$ is given by the vector potential mKdV equation

$$
\boldsymbol{k}_{t}=\boldsymbol{k}_{x x x}+\frac{3}{2}\left(\left(k_{x}{ }^{2}+\left|\boldsymbol{k}_{x}\right|^{2}\right) \boldsymbol{k}\right)_{x}
$$

up to a convective term, while the -1 flow on $\boldsymbol{k}$ reduces to the vector SG equation

$$
\left(k^{-1} \boldsymbol{k}_{t}\right)_{x}=-\chi \boldsymbol{k}
$$

after a conformal scaling on $t$ is used to put $\hat{n}=\vec{h}_{\perp}$. All these flows inherit a natural bi-Hamiltonian formulation which is obtained by splitting the operators $\mathcal{I}, \mathcal{H}$ relative to $\hat{n}$.

Theorem 8: The flow equation on the unconstrained variable $\boldsymbol{k}(t, x)$ takes the form

$$
\boldsymbol{k}_{t}=\chi \mathcal{I}^{\perp}\left(\boldsymbol{h}^{\perp}\right)+\mathcal{H}^{\perp}\left(\boldsymbol{h}^{\perp}\right)=\chi \varpi^{\perp}+\varpi^{\perp}
$$

given by

$$
\left.\mathcal{I}^{\perp}=k D_{x}^{-1}\left(k+\boldsymbol{k}\left(k^{-1} \boldsymbol{k} \cdot\right)\right)-\boldsymbol{k}\right\lrcorner D_{x}^{-1}(\boldsymbol{k} \wedge)
$$

and

$$
\mathcal{H}^{\perp}=D_{x}+D_{x}\left(\boldsymbol{k} D_{x}^{-1}\left(\left(\boldsymbol{k}_{x} \cdot\right)-k^{-1} k_{x}(\boldsymbol{k} \cdot)\right)\right) .
$$

These operators $\mathcal{I}^{\perp}, \mathcal{H}^{\perp}$ are a compatible Hamiltonian pair with respect to $\boldsymbol{k}$, where $\boldsymbol{h}^{\perp}=\vec{h}_{\perp}-\hat{n} \cdot \vec{h}_{\perp} \hat{n}, \varpi^{\perp}=\vec{\varpi}-\hat{n} \cdot \vec{\varpi} \hat{n}$ (and likewise for $\varpi^{\prime}{ }^{\perp}$ ) are Hamiltonian covector and vector fields, respectively.

In summary: In any $n$-dimensional constant-curvature space $(N, g)$ there is a hierarchy of bi-Hamiltonian flows of non-stretching curves $\gamma(t, x)$, where the 0 flow is described by the mKdV map equation (4.8) and the $+k$ flow is a higher-order analog, while the wave map equation (4.11) describes a -1 flow that is annihilated by the recursion operator of the hierarchy. In a parallel frame the principal normal components along $\gamma$ for these flows respectively satisfy a vector mKdV equation (4.7) and a vector hyperbolic equation (4.9). A covariantly-constant frame gives rise to potentials for the principal normal components along $\gamma$, satisfying a vector potential mKdV equation (4.21) and a vector SG equation (4.22), respectively.

\subsection{Hamiltonian operators in Lie group spaces}

Importantly, the idea of utilizing a non-adapted moving frame for non-stretching curve flows will now be carried over to Riemannian spaces $(N, g)$ without the property of constant curvature, specifically compact semisimple Lie group manifolds $N \simeq \mathcal{G}$. These spaces have a natural Riemannian structure [21] which is invariant under leftmultiplication, with the Riemannian metric $g$ given by the bilinear Cartan-Killing form in the Lie algebra $\mathfrak{g}$ of the Lie group $\mathcal{G}$. (Recall the Cartan-Killing form is nondegenerate when and only when $\mathcal{G}$ is semisimple, and positive when and only when $\mathcal{G}$ is compact.) To proceed, let $(N \simeq \mathcal{G}, g)$ be a $n$-dimensional (compact semisimple) Lie group manifold $(n>2)$, generalizing the three-dimensional case $N=S U(2) \simeq S^{3}$ (cf. section 3.3). Any 
orthonormal basis for the Lie algebra $\mathfrak{g} \simeq T_{x} N$ provides a left-invariant orthonormal frame $e_{a}$ on $N$ whose local structure group is $\mathcal{G}$ itself, satisfying the commutator property

$$
\left[e_{a}, e_{b}\right]=c_{a b}{ }^{c} e_{c}
$$

where $c_{a b}{ }^{c}=c_{[a b]}{ }^{c}$ denotes the Lie algebra structure constants. Note, the Jacobi relation is $c_{[a b}{ }^{e} c_{c] d e}=0$ while semisimplicity is expressed by $c_{a b c}=c_{[a b c]}$, where indices are raised and lowered by the Cartan-Killing form $g\left(e_{a}, e_{b}\right)=-\frac{1}{2} c_{a c}{ }^{d} c_{b d}{ }^{c}=\delta_{a b}$. The frame curvature matrix for $N$ is given by the algebraic expression

$$
R\left(e_{c}, e_{d}\right)_{a}{ }^{b}=\frac{1}{4} c_{c d}{ }^{e} c_{a e}{ }^{b} .
$$

A distinguishing feature of a left-invariant frame is that this matrix is constant on $N$.

The Cartan structure equations associated to the flow of a curve $\gamma(t, x)$ generalize from three dimensions, i.e. $N=S U(2)$, to $n$ dimensions, i.e. $N=\mathcal{G}$, with the structure constants $c_{a b}{ }^{c}$ in place of $\epsilon_{a b}{ }^{c}$ and with the Lie-algebra bracket in place of a vector cross product. In particular, let $\operatorname{ad}(q)_{a}{ }^{b}=c_{c a}{ }^{b} q^{c}$ denote the bracket on the $n$-component vector $q^{a}$. Note $q^{a}$ has the geometrical meaning of a potential for the components $v^{a}=q_{x}^{a}$ of the principal normal along $\gamma$. Hereafter it will be convenient to scale out a constant factor $\chi$ from the curvature $R\left(e_{c}, e_{d}\right)_{a}{ }^{b} \rightarrow \chi c_{c d}{ }^{e} c_{a e}{ }^{b}$ by putting $c_{a b}{ }^{c} \rightarrow 2 \sqrt{\chi} c_{a b}{ }^{c}$ for ease of comparison with the three dimensional case.

Thus the flow equation on $\vec{q}$ (in index-free notation) is given by

$$
\vec{q}_{t}=\sqrt{\chi} \mathcal{I}\left(\vec{h}_{\perp}\right)+\mathcal{H}^{\prime}\left(\vec{h}_{\perp}\right)=\sqrt{\chi} \vec{\varpi}^{\prime}+\vec{\varpi}
$$

where

$$
\mathcal{I}=\operatorname{ad}(q)
$$

is an algebraic operator and

$$
\mathcal{H}^{\prime}=\mathcal{D}_{x}+\vec{q}_{x} D_{x}^{-1}\left(\vec{q}_{x} \cdot\right)+\vec{q}\left(\vec{q}_{x} \cdot\right)=\mathcal{H}+\sqrt{\chi} \mathcal{I}
$$

is a covariant version of the previous operator $\mathcal{H}=D_{x}+\vec{q}_{x} D_{x}^{-1}\left(\vec{q}_{x} \cdot\right)+\vec{q}\left(\vec{q}_{x} \cdot\right)$ using the derivative

$$
\mathcal{D}_{x}=D_{x}+\sqrt{\chi} \operatorname{ad}(q) .
$$

As in three dimensions, the operators $\mathcal{H}\left(\right.$ or $\mathcal{H}^{\prime}$ ) and $\mathcal{I}$ are a compatible Hamiltonian pair with respect to the constrained variable $\vec{q}(t, x)$, on the domain of $x$-jet space coordinates $\perp \vec{q}$. So $\vec{\varpi}$ and $\vec{\varpi}^{\prime}$ represent Hamiltonian vector fields, while $\vec{h}_{\perp}$ represents a covector field. However in higher than three dimensions the operator $\mathcal{I}$ is no longer invertible because of the fact that for any vector $\vec{q}$ in $\mathfrak{g}$ the kernel of $\operatorname{ad}(q)$ has a (nonzero) dimension at least equal to the rank of the Lie algebra $\mathfrak{g}$, and all semisimple Lie algebras of dimension $n>3$ have rank greater than 1 (see e.g. [28]). Thus in the $n>3$ dimensional case, $\mathcal{I}^{-1}$ does not exist since $\mathcal{I}(\vec{c})=0$ for at least one nonzero vector $\vec{c}$ linearly independent of $\vec{q}$ in $\mathfrak{g}$. This difficulty can be overcome by restricting the operators $\mathcal{H}, \mathcal{I}$ to a suitable smaller domain, which will be seen to necessarily introduce some nonlocality. 
Let $C_{q}$ denote the centralizer of $\vec{q}$ in $\mathfrak{g}$, namely the set of all vectors $\vec{c}$ commuting with $\vec{q}$ in $\mathfrak{g}$,

$$
\operatorname{ad}(q) \vec{c}=0 .
$$

Note that, for a given vector $\vec{q}$, the centralizer $C_{q}$ is algebraically determined through the Lie algebra structure constants. Write $C_{q}^{\perp}$ for the orthogonal complement of $C_{q}$ in $\mathfrak{g}$ with respect to the Cartan-Killing metric, so $\mathfrak{g}=C_{q} \oplus C_{q}^{\perp}$, and write $C_{\perp q}$ for the orthogonal complement with respect to $\vec{q}$ in $C_{q}$. The sets $C_{q}, C_{\perp q}$ are Lie subalgebras of $\mathfrak{g}$, and the set $C_{q}^{\perp}$ is an invariant vector subspace in $\mathfrak{g}$ under $\operatorname{ad}(q)$. Let $\mathcal{P}_{C_{\perp q}}, \mathcal{P}_{C_{q}}, \mathcal{P}_{C_{q}}^{\perp}$ denote the projection operators in $\mathfrak{g}$ onto $C_{\perp q}, C_{q}, C_{q}^{\perp}$. Now decompose $\vec{h}_{\perp}$ into the orthogonal parts $\vec{h}_{C}=\mathcal{P}_{C_{\perp q}}\left(\vec{h}_{\perp}\right), \vec{h}_{C^{\perp}}=\mathcal{P}_{C_{q}}^{\perp}\left(\vec{h}_{\perp}\right)$.

Proposition 9: For all vectors $\vec{c}$ in $C_{\perp q}, \vec{c} \cdot \mathcal{I}=0$ while $\vec{c} \cdot \mathcal{H}=-\vec{c}_{x} \cdot \mathcal{P}_{C_{q}}^{\perp}-\vec{c}_{x}$. $\vec{q} D_{x}^{-1}\left(\vec{q}_{x} \cdot\right)+\left(\vec{c} \cdot D_{x}\right) \mathcal{P}_{C_{\perp q}}$.

Hence $\mathcal{I}$ restricts to an operator in $C_{q}^{\perp}$ acting on $\vec{h}_{C^{\perp}}$ by

$$
\mathcal{I}\left(\vec{h}_{\perp}\right)=\operatorname{ad}(q) \vec{h}_{C^{\perp}}:=\mathcal{I}_{\perp}\left(\vec{h}_{C^{\perp}}\right) .
$$

Consequently, $\mathcal{I}_{\perp}$ is now invertible on $\vec{h}_{C^{\perp}}$, with

$$
\mathcal{I}_{\perp}^{-1}=\operatorname{ad}(q)_{\perp}^{-1}
$$

where $\operatorname{ad}()_{\perp}$ denotes the restriction of $\operatorname{ad}()$ to $C_{q}^{\perp}$. Moreover, $\mathcal{H}$ can be adjusted to yield an operator in $C_{q}^{\perp}$ if the condition

$$
\vec{c} \cdot \mathcal{H}\left(\vec{h}_{\perp}\right)=0
$$

for all vectors $\vec{c}$ in $C_{\perp q}$ is used to determine $\vec{h}_{C}$ in terms of $\vec{h}_{C^{\perp}}$ as follows: Introduce an orthonormal basis $\vec{c}^{\mu}$ for the vector space $C_{\perp q}$, so

$$
\vec{c}^{\mu} \cdot \vec{c}^{\nu}=\delta^{\mu \nu},
$$

and fix the $x$ dependence of the basis by the orthogonality property

$$
\vec{c}^{\mu} \cdot \vec{c}_{x}^{\nu}=0 .
$$

Then the basis coefficients of the condition (4.35) on $\mathcal{H}$ yield the equation

$$
D_{x} h_{\mu}=h_{\|} \vec{c}^{\mu} \cdot \vec{q}_{x}+\vec{c}_{x}^{\mu} \cdot \vec{h}_{C^{\perp}}
$$

with

$$
D_{x} h_{\|}=\vec{q}_{x} \cdot \vec{h}_{C^{\perp}}+h_{\mu} \vec{c}^{\mu} \cdot \vec{q}_{x}
$$

where $h_{\mu}=\vec{h}_{C} \cdot \vec{c}^{\mu}$ denotes the basis coefficients of $\vec{h}_{C}$. Hence $\mathcal{H}$ becomes an operator in $C_{q}^{\perp}$ acting on $\vec{h}_{C^{\perp}}$ by

$$
\mathcal{H}\left(\vec{h}_{\perp}\right)=D_{x}\left(\vec{h}_{C^{\perp}}+h_{\|} \vec{q}+h_{\mu} \vec{c}^{\mu}\right)=D_{x}^{\perp} \vec{h}_{C^{\perp}}+h_{\|} \vec{q}_{x}^{\perp}+h_{\mu} \vec{c}_{x}^{\mu}:=\mathcal{H}_{\perp}\left(\vec{h}_{C^{\perp}}\right)(4
$$

where

$$
D_{x}^{\perp}=\mathcal{P}_{C_{q}}^{\perp}\left(D_{x}\right)=D_{x}+\vec{q}\left(\vec{q}_{x} \cdot\right)+\vec{c}_{\mu} \vec{c}_{x}^{\mu}
$$


and

$$
\vec{q}_{x}^{\perp}=\mathcal{P}_{C_{q}}^{\perp}\left(\vec{q}_{x}\right)=\vec{q}_{x}+\vec{c}_{\mu} \vec{q} \cdot \vec{c}_{x}^{\mu} .
$$

To obtain the closest generalization of the results in three dimensions, $\vec{q}$ should be restricted to obey the condition

$$
\mathcal{P}_{C_{q}}\left(\vec{q}_{x}\right)=0
$$

so thus $\vec{q}_{x}=\vec{q}_{x}^{\perp}$ will lie in the range of the operators $\mathcal{H}_{\perp}, \mathcal{I}_{\perp}$. In this situation, $h_{\|}$and $h_{\mu}$ are able to be explicitly determined in terms of $\vec{h}_{C^{\perp}}$ by

$$
D_{x} h_{\|}=\vec{q}_{x} \cdot \vec{h}_{C^{\perp}}, \quad D_{x} h_{\mu}=\vec{c}_{x}^{\mu} \cdot \vec{h}_{C^{\perp}} .
$$

In addition, we have

$$
\vec{c}_{x}^{\mu}=\operatorname{ad}(q)_{\perp}^{-1}\left[\vec{c}^{\mu}, \vec{q}_{x}\right]
$$

where the bracket denotes the Lie-algebra product.

The following result is a generalization of theorem $5: \mathcal{I}_{\perp}=\operatorname{ad}(q)_{\perp}$ and $\mathcal{H}_{\perp}=$ $D_{x}^{\perp}+\vec{q}_{x} D_{x}^{-1}\left(\vec{q}_{x} \cdot\right)+\vec{c}_{x}^{\mu} D_{x}^{-1}\left(\vec{c}_{x}^{\mu} \cdot\right)$ are a pair of compatible Hamiltonian (cosymplectic) operators obeying $\mathcal{P}_{C_{\perp q}}\left(\mathcal{I}_{\perp}\right)=\mathcal{P}_{C_{\perp q}}\left(\mathcal{H}_{\perp}\right)=0$ with respect to the constrained variable $\vec{q}(t, x)$, and $\mathcal{J}_{\perp}=\mathcal{I}_{\perp}^{-1}=\operatorname{ad}(q)_{\perp}^{-1}$ is a symplectic operator (the domains of these operators consists of the $x$-jet space coordinates associated to $\left.C_{q}^{\perp}\right)$. The flow equation on $\vec{q}$ is given by $\vec{q}_{t}=\sqrt{\chi} \vec{\varpi}_{C^{\perp}}^{\prime}+\vec{\varpi}_{C^{\perp}}$ where $\vec{\varpi}_{C^{\perp}}=\mathcal{H}_{\perp}^{\prime}\left(\vec{h}_{C^{\perp}}\right), \vec{\varpi}_{C^{\perp}}^{\prime}=\mathcal{I}_{\perp}\left(\vec{h}_{C^{\perp}}\right)$ are Hamiltonian vector fields produced from the covector field $\vec{h}_{C^{\perp}}$. Here

$$
\mathcal{H}_{\perp}^{\prime}=\mathcal{D}_{x}^{\perp}+\vec{q}_{x} D_{x}^{-1}\left(\vec{q}_{x} \cdot\right)+\vec{c}_{x}^{\mu} D_{x}^{-1}\left(\vec{c}_{x}^{\mu} \cdot\right)=\mathcal{H}_{\perp}+\sqrt{\chi} \mathcal{I}_{\perp}
$$

is a covariant version of $\mathcal{H}_{\perp}$ with

$$
\mathcal{D}_{x}^{\perp}=D_{x}^{\perp}+\sqrt{\chi} \operatorname{ad}(q)_{\perp} .
$$

Moreover $\mathcal{R}^{\prime}=\mathcal{H}_{\perp}^{\prime} \circ \mathcal{J}_{\perp}$ is a hereditary recursion operator.

Remark: $N=S U(2) \simeq S^{3}$ is the only compact semisimple Lie group of dimension $1<n \leq 3$. For this group, the Lie bracket $\operatorname{ad}(q)$ has an empty kernel for any (nonzero) vector $\vec{q}$ in the Lie algebra $\mathfrak{g}=\mathfrak{s u}(2) \simeq \mathfrak{s o}(3)$ and hence the variables $\vec{c}^{\mu}$ disappear. Consequently, on the domain of $x$-jet space coordinates $\perp \vec{q}$, the operators $\mathcal{H}, \mathcal{I}$ are a compatible Hamiltonian pair such that the inverse operator $\mathcal{J}=\mathcal{I}^{-1}$ exists and is a Hamiltonian symplectic operator, and $\mathcal{R}=\mathcal{H} \circ \mathcal{J}$ is a hereditary recursion operator, agreeing with the operators in theorem 5 .

As in three dimensions, $\mathcal{R}^{\prime}$ generates a hierarchy of bi-Hamiltonian flows. At the bottom the 0 flow is simply

$$
\vec{\varpi}_{C^{\perp}}^{\prime}=\vec{q}_{x}
$$

while next the +1 flow is given by

$$
\vec{\varpi}_{C^{\perp}}^{\prime}=\mathcal{R}^{\prime}\left(\vec{q}_{x}\right)=\mathcal{D}_{x}\left(\operatorname{ad}(q)_{\perp}^{-1} \vec{q}_{x}+D_{x}^{-1}\left(\vec{c}_{x}^{\mu} \cdot \operatorname{ad}(q)_{\perp}^{-1} \vec{q}_{x}\right) \vec{c}_{\mu}\right)
$$


which involves essential nonlocal terms in ( $x$ derivatives of $)$ the variables $\vec{c}^{\mu}$. Accordingly, modulo such nonlocal terms, the 0 flow equation describes a natural generalization of the $S O(3)$ group-motion equation on $\vec{q}$ for the Lie group $\mathcal{G}$,

$$
\left(\vec{q}_{t}\right)_{\text {local }}=D_{x}^{\perp}\left(\operatorname{ad}(q)_{\perp}^{-1} \vec{q}_{x}\right)+2 \sqrt{\chi} \vec{q}_{x} .
$$

Under this flow the curve $\gamma$ undergoes a non-stretching motion given by the geometric map equation

$$
\left(\gamma_{t}\right)_{\perp}=\operatorname{ad}\left(\gamma_{x}\right)_{\perp}^{-1} \nabla_{x} \gamma_{x}
$$

with $\left|\gamma_{x}\right|_{g}=1$, where $\perp$ denotes the projection with respect to $\mathcal{P}_{C_{q}}^{\perp}$ in the tangent space $T_{x} N \simeq \mathfrak{g}$ of the curve $\gamma$, corresponding to

$$
\vec{h}_{C^{\perp}}=\operatorname{ad}(q)_{\perp}^{-1} \vec{q}_{x} .
$$

In a similar way the +1 flow equation describes a linear combination of the group-motion equation (4.50) and a group-invariant generalization of the mKdV equation on $\vec{q}$, given by

$$
\left(\vec{q}_{t}\right)_{\text {local }}=D_{x}^{\perp}\left(\operatorname{ad}(q)_{\perp}^{-1}\left(D_{x}^{\perp}\left(\operatorname{ad}(q)_{\perp}^{-1} \vec{q}_{x}\right)\right)\right)-\frac{1}{2}\left(\left|\operatorname{ad}(q)_{\perp}^{-1} \vec{q}_{x}\right|^{2} \vec{q}\right)_{x}
$$

up to nonlocal terms. The curve motion of $\gamma$ produced by this flow is an analogous groupinvariant $\mathrm{mKdV}$ map equation which contains essential nonlocal terms, corresponding to

$$
\left(\vec{h}_{C^{\perp}}\right)_{\text {local }}=\operatorname{ad}(q)_{\perp}^{-1}\left(\mathcal{D}_{x}\left(\operatorname{ad}(q)_{\perp}^{-1} \vec{q}_{x}\right)\right) .
$$

Higher order (even and odd) flows are similar in form to the $0,+1$ flows.

There is a -1 flow that is mapped into $\vec{\varpi}=0$ under $\mathcal{R}^{\prime}$ in the hierarchy. In this flow $\vec{q}$ and $\vec{h}_{C^{\perp}}$ satisfy the equations

$$
0=\mathcal{D}_{t} \vec{q}=\mathcal{D}_{x} \vec{h}
$$

which are equivalent to a constrained vector chiral model, where $\vec{h}=\vec{h}_{C^{\perp}}+h_{\|} \vec{q}+h_{\mu} \vec{c}^{\mu}$ obeys the conservation law $0=D_{x}|\vec{h}|^{2}$ and so $|\vec{h}|^{2}=1$ after $t$ is conformally scaled. Another formulation of the -1 flow equation is obtained by substituting

$$
\vec{h}_{C^{\perp}}=\operatorname{ad}(q)_{\perp}^{-1} \vec{q}_{t}
$$

from the first equation into the second equation, which then yields a vector hyperbolic equation on $\vec{q}(t, x)$,

$$
\left(\mathcal{D}_{x} \vec{q}_{t}\right)_{\text {local }}=\left[\vec{q}_{x}, \operatorname{ad}(q)_{\perp}^{-1} \vec{q}_{x}\right]
$$

up to certain nonlocal terms involving $x$ derivatives of $\vec{c}^{\mu}$. The corresponding curve motion of $\gamma$ is simply given by the non-stretching wave map equation

$$
\nabla_{t} \gamma_{x}=0, \quad 1=\left|\gamma_{x}\right|_{g}=\left|\gamma_{t}\right|_{g}
$$

In summary: In $n$-dimensional Lie-group spaces $(N \simeq \mathcal{G}, g)$ there is a hierarchy of bi-Hamiltonian flows of non-stretching curves $\gamma(t, x)$, subject to the geometric condition that the principal normal along $\gamma$ is orthogonal to the Lie-algebra centralizer in the 
normal space to $\gamma$, i.e. $g\left(\nabla_{x} \gamma_{x}, \Gamma\right)=0$ for all $\Gamma$ annihilated by $\operatorname{ad}\left(\gamma_{x}\right)$. The hierarchy starts at the 0 flow whose normal motion is described by the geometric group-motion map equation (4.51) associated to $\mathcal{G}$, while the wave map equation (4.58) describes a -1 flow that is annihilated by the recursion operator of the hierarchy. In a left-invariant frame the principal normal components along $\gamma$ for these flows respectively satisfy a vector group-motion equation (4.50) and a vector hyperbolic equation (4.57).

\section{Conclusion}

The main goal of this paper has been the study of various kinds of moving frames parallel, covariantly-constant, left invariant — in deriving bi-Hamiltonian operators and vector soliton equations from flows of non-stretching curves in Riemannian manifolds. A main insight, following the ideas of Ref. [8, 9, 11, is that the bi-Hamiltonian structure is geometrically encoded in the Cartan structure equations for the torsion and curvature associated to a curve flow whenever the frame curve matrix is everywhere constant on the manifold. The curve motions corresponding to the soliton equations determined by such a bi-Hamiltonian structure are found to be geometric map equations, in particular wave maps and $\mathrm{mKdV}$ /group-invariant analogs of Schrödinger maps.

A unified treatment of constant-curvature manifolds and Lie-group manifolds based on Riemannian symmetric spaces will be given in a forthcoming paper.

\section{Acknowledgments}

The author is supported by an N.S.E.R.C. grant. Jing Ping Wang is thanked for very fruitful discussions in the early stage of this research. The referees are thanked for comments which have improved the paper.

\section{Appendix}

The following summary of Hamiltonian structure is adapted from Ref. [19, 20].

Consider the jet space $\left\{\left(x, u, u_{x}, u_{x x}, \ldots\right)\right\}$ of a scalar or vector variable $u(x)$. Let $h \cdot \partial / \partial u$ be a vector field with component $(\mathrm{s}) h=h\left(x, u, u_{x}, u_{x x}, \ldots\right)$, and $\varpi \cdot d u$ a covector field with component(s) $\varpi=\varpi\left(x, u, u_{x}, u_{x x}, \ldots\right)$, where a dot denotes summation over any components. Write $\delta_{h}=\operatorname{pr}(h \partial / \partial u)$ denoting the variation (linearization) induced by $h$, i.e. the prolonged action of the vector field; write $\delta / \delta u$ for the variational derivative with respect to $u$. Let $\theta$ denote a vertical uni-vector [20] dual to $d u$, i.e. $\theta\lrcorner d u=1$.

A skew-adjoint operator $\mathcal{H}$ mapping $\varpi$ into $h$ is Hamiltonian (cosymplectic) iff

$$
0=\int\left(\theta \wedge \delta_{h} \mathcal{H}(\theta)\right) d x \quad \text { for } h=\mathcal{H}(\theta) .
$$

This condition is equivalent to the vanishing of the Schouten bracket of $\mathcal{H}$. A skew- 
adjoint operator $\mathcal{J}$ mapping $h$ into $\varpi$ is symplectic iff

$$
0=\int\left(h_{3} \cdot \delta_{h_{1}} \mathcal{J}\left(h_{2}\right)+\text { cyclic }\right) d x
$$

for arbitrary $h_{i}$. The operator $\mathcal{R}=\mathcal{H} \circ \mathcal{J}$ is a hereditary recursion operator on $h$ iff

$$
0=\mathcal{R}\left(\delta_{h_{1}} \mathcal{R}\left(h_{2}\right)-\delta_{h_{2}} \mathcal{R}\left(h_{1}\right)\right)
$$

for arbitrary $h_{i}$.

A (Hamiltonian) functional is an expression $\mathfrak{H}=\int H\left(x, u, u_{x}, u_{x x}, \ldots\right) d x$. The Poisson bracket with respect to a Hamiltonian operator $\mathcal{H}$ is defined by

$$
\{\mathfrak{H}, \mathfrak{E}\}_{\mathcal{H}}=\int(\mathcal{H}(\delta \mathfrak{E} / \delta u) \cdot \delta \mathfrak{H} / \delta u) d x
$$

for any functionals $\mathfrak{H}, \mathfrak{E}$. This bracket is skew and obeys the Jacobi identity. $h \cdot \partial / \partial u$ is a Hamiltonian vector field if there exists a (Hamiltonian) functional $\mathfrak{H}$ such that

$$
\delta_{h} \mathfrak{E}=\{\mathfrak{H}, \mathfrak{E}\}_{\mathcal{H}}
$$

holding for all functionals $\mathfrak{E}$. $\varpi \cdot d u$ is a variational (Hamiltonian) covector field if there exists a (Hamiltonian) functional $\mathfrak{H}$ such that

$$
\varpi=\delta \mathfrak{H} / \delta u
$$

There is a canonical pairing between Hamiltonian vector fields $h \cdot \partial / \partial u$ and variational covector fields $\varpi \cdot d u$ via

$$
\varpi=\omega(h, \cdot)_{\mathcal{J}}
$$

where

$$
\omega\left(h_{1}, h_{2}\right)_{\mathcal{J}}=\int h_{1} \cdot \mathcal{J}\left(h_{2}\right) d x
$$

is a symplectic 2 -form. In particular, the operators $\mathcal{J}$ and $\mathcal{H}$ directly give mappings $h \cdot \partial / \partial u \mapsto \mathcal{J}(h) \cdot d u$ and $\varpi \cdot d u \mapsto \mathcal{H}(\varpi) \cdot \partial / \partial u$.

\section{References}

[1] M. Lakshmanan, J. Math. Phys. 20, 1667-1672 (1979).

[2] R.L. Ricca, Nature 352, 561-562, (1991).

[3] H. Hasimoto, J. Fluid Mech. 51, 477-485 (1972).

[4] N.-H. Chang, J. Shatah, K. Uhlenbeck, Comm. Pure Applied Math. 53, 590-602 (2000).

[5] K. Polhmeyer, Comm. Math. Phys. 46, 207-221 (1976).

[6] G.L. Lamb Jr., J. Math. Phys. 18, 1654-1661 (1977).

[7] A. Doliwa, P.M. Santini, Phys. Lett. A 185, 373-384 (1994).

[8] G. Marí Beffa, J. Sanders, J.-P. Wang, J. Nonlinear Sci. 12, 143-167 (2002).

[9] J. Sanders, J.-P. Wang, Moscow Mathematical Journal 3, 1369-1393 (2003).

[10] R. Bishop, Amer. Math. Monthly 82, 246-251 (1975). 
[11] S.C. Anco, J.-P. Wang, in preparation (2005).

[12] J.-P. Wang, in Symmetry and Perturbation Theory, eds. S. Abenda, G. Gaeta, S. Walcher (World Scientific 2003).

[13] H.W. Guggenheimer, Differential Geometry (McGraw Hill 1963).

[14] J. Sanders, J.-P. Wang, J. Difference Equ. Appl. 12, 983-995 (2006).

[15] K.-S. Chou, C. Qu, Physica D 162, 9-33 (2002).

[16] K.-S. Chou, C. Qu, Chaos, Solitons and Fractals 14, 29-44 (2002).

[17] K.-S. Chou, C. Qu, J. Nonlinear Sci. 13, 487-517 (2003).

[18] K.-S. Chou, C. Qu, Chaos, Solitons and Fractals 19, 47-53 (2004).

[19] I. Dorfman, Dirac Structures and Integrability of Nonlinear Evolution Equations (Wiley 1993).

[20] P.J. Olver, Applications of Lie Groups to Differential Equations (Springer, New York 1986).

[21] S. Kobayashi, K. Nomizu, Foundations of Differential Geometry Volumes I and II, (Wiley 1969).

[22] G. Marí Beffa, Contemp. Math. 285, 29-38 (2001).

[23] V.V. Sokolov and T. Wolf, J. Phys. A: Math. and Gen. 34, 11139-11148 (2001).

[24] S. Anco, T. Wolf, J. Nonlinear Math. Phys. 12, 13-31 (2005); ibid. J. Nonlinear Math. Phys. 12, 607-608 (2005).

[25] I. Bakas, Q.-H. Park, H.-J. Shin, Phys. Lett. B 372, 45-52 (1996).

[26] K. Pohlmeyer, K.-H. Rehren, J. Math. Phys. 20, 2628-2632 (1979).

[27] V.E. Zakharov, A.V. Mikailov, Sov. Phys. JETP 47, 1017-1027 (1978).

[28] A. Sagle and R. Walde, Introduction to Lie Groups and Lie Algebras, (Academic Press 1973). 